\title{
Time-Course of Metabolic and Proteomic Responses to Different Nitrate/Ammonium Availabilities in Roots and Leaves of Maize
}

\author{
Bhakti Prinsi * (i) and Luca Espen (10) \\ Department of Agricultural and Environmental Sciences-Production, Landscape, Agroenergy (DiSAA), \\ Università degli Studi di Milano, Via Celoria 2, 20133 Milano, Italy; luca.espen@unimi.it \\ * Correspondence: bhakti.prinsi@unimi.it; Tel.: +39-02-503-16610
}

Received: 13 June 2018; Accepted: 25 July 2018; Published: 27 July 2018

\begin{abstract}
The availability of nitrate and ammonium significantly affects plant growth. Co-provision of both nutrients is generally the best nutritional condition, due to metabolic interactions not yet fully elucidated. In this study, maize grown in hydroponics was exposed to different nitrogen $(\mathrm{N})$ availabilities, consisting of nitrate, ammonium and co-provision. Roots and leaves were analyzed after 6,30 , and $54 \mathrm{~h}$ by biochemical evaluations and proteomics. The ammonium-fed plants showed the lowest biomass accumulation and the lowest ratio of inorganic to organic $\mathrm{N}$ content, suggesting a metabolic need to assimilate ammonium that was not evident in plants grown in co-provision. The $\mathrm{N}$ sources differently affected the root proteome, inducing changes in abundance of proteins involved in $\mathrm{N}$ and carbon (C) metabolisms, cell water homeostasis, and cell wall metabolism. Notable among these changes was that some root enzymes, such as asparagine synthetase, phosphoenolpyruvate (PEP) carboxylase, and formate dehydrogenase showed a relevant upsurge only under the sole ammonium nutrition. However, the leaf proteome appeared mainly influenced by total $\mathrm{N}$ availability, showing changes in the abundance of several proteins involved in photosynthesis and in energy metabolism. Overall, the study provides novel information about the biochemical determinants involved in plant adaptation to different $\mathrm{N}$ mineral forms.
\end{abstract}

Keywords: ammonium; co-provision; maize; nitrate; plant nutrition; proteomics

\section{Introduction}

Nitrogen $(\mathrm{N})$ is the mineral element required in the highest amount by plants: nitrate $\left(\mathrm{NO}_{3}{ }^{-}\right)$ and ammonium $\left(\mathrm{NH}_{4}^{+}\right)$represent the main inorganic $\mathrm{N}$ sources [1]. Since the proportion in which $\mathrm{NO}_{3}{ }^{-}$and $\mathrm{NH}_{4}{ }^{+}$are available in agricultural soils strongly affects crop productivity [2], a better understanding of plant biochemical responses to $\mathrm{N}$ sources could help to improve agricultural sustainability. Nitrate and $\mathrm{NH}_{4}{ }^{+}$have different, and sometimes opposite, effects on plant development, growth rate, root architecture, and leaf expansion [3]. The use of $\mathrm{NO}_{3}{ }^{-}$and $\mathrm{NH}_{4}{ }^{+}$by plants is sustained by different mechanisms of acquisition, allocation, and assimilation [2,4,5]. After uptake by roots, $\mathrm{NO}_{3}{ }^{-}$is firstly reduced by nitrate reductase (NR) and nitrite reductase (NiR) to generate $\mathrm{NH}_{4}{ }^{+}$, which, together with the quota derived from soil and metabolism, is assimilated into amino acids by the glutamine synthetase/glutamate synthase pathway (GS/GOGAT) [2]. The contribution of roots and leaves in $\mathrm{N}$ assimilation is influenced by several factors, but in both cases, the process involves several interactions with carbon (C) metabolism that allow the plant to sustain the requirements of $C$ skeletons and of metabolic energy [6]. The use of $\mathrm{NO}_{3}{ }^{-}$or $\mathrm{NH}_{4}{ }^{+}$by plants is associated with different balancing among glycolysis, the oxidative pentose pathway, and the tricarboxylic acid (TCA) cycle [2,7]. Considering the theoretical metabolic costs [8] and the demand for reducing equivalents [9], the use of 
$\mathrm{NH}_{4}{ }^{+}$by plants seems to be advantageous compared to that of $\mathrm{NO}_{3}{ }^{-}$, but this prediction does not often coincide with empirical observations [3]. An exclusive, or excessive, $\mathrm{NH}_{4}{ }^{+}$nutrition could have adverse impacts on plants, including alterations in root metabolism, plant ionic imbalances, and foliar oxidative stress $[10,11]$. Plant responses also significantly depend on the relative proportion between the $\mathrm{NH}_{4}{ }^{+}$and the $\mathrm{NO}_{3}{ }^{-}$available in the soil. The co-provision of both $\mathrm{NH}_{4}{ }^{+}$and $\mathrm{NO}_{3}{ }^{-}$is generally considered the optimal $\mathrm{N}$ condition, in which the two nutrients reveal synergistic beneficial effects [12]. The synergy mainly arises from the reciprocal influences between $\mathrm{NH}_{4}{ }^{+}$and $\mathrm{NO}_{3}{ }^{-}$on their uptake, on root morphology, on the transport of $\mathrm{N}$ compounds from roots to shoots, and on plant $\mathrm{C}$ metabolism. Overall, these interactions improve the capabilities of the plants for $\mathrm{N}$ acquisition and assimilation [13]. Large-scale approaches have turned out to be very useful to investigate the complexity of N nutrition in plants, as proven by many transcriptomic studies conducted in Arabidopsis thaliana [11,14]. However, several aspects have yet to be fully elucidated, such as the interactions and communications between $\mathrm{NO}_{3}{ }^{-}$and $\mathrm{NH}_{4}{ }^{+}$and between roots and leaves.

Maize (Zea mays L.) is a crop of worldwide economic relevance, and is characterized by C4 metabolism and a very high demand for $\mathrm{N}$ inputs in agricultural systems [15]. Some large-scale studies have been devoted to investigating $\mathrm{NO}_{3}{ }^{-}$metabolism in maize, providing new evidence that this anion acts as a signal influencing its uptake and assimilation both at transcriptional and protein levels [16-18]. Moreover, these studies have been useful in revealing new aspects regarding the interlinks between $\mathrm{C}$ and $\mathrm{N}$ metabolism in plants $[19,20]$. In particular, comparative proteomics shows that $\mathrm{NO}_{3}{ }^{-}$availability evokes different responses in roots and leaves, highlighting the importance of analyzing both organs [20]. To our knowledge, similar approaches have not yet been applied in maize to study the responses to $\mathrm{NH}_{4}{ }^{+}$, either as sole $\mathrm{N}$ nutrient or in combination with $\mathrm{NO}_{3}{ }^{-}$.

In this study, maize plants were exposed to $\mathrm{NO}_{3}{ }^{-}$, to $\mathrm{NH}_{4}{ }^{+}$, or to co-provision and analyzed over a period of three days, in order to appreciate plant metabolic and biochemical differences. The evaluation of plant growth and nutritional status in roots and leaves was combined with a comparative proteomic approach. This investigation showed that $\mathrm{NO}_{3}{ }^{-}$and $\mathrm{NH}_{4}{ }^{+}$had different effects on plant growth and that the availability of $\mathrm{NO}_{3}{ }^{-}$in co-provision affected the accumulation and assimilation of $\mathrm{NH}_{4}{ }^{+}$in roots and leaves. Interestingly, the root proteome was more specifically influenced by the $\mathrm{N}$ source, while leaf profiles were mainly affected by the total $\mathrm{N}$ availability. Moreover, some proteomic changes were specifically induced by $\mathrm{NH}_{4}{ }^{+}$as sole nutrient and absent in co-provision or with $\mathrm{NO}_{3}{ }^{-}$nutrition. Taken together, the results suggest that $\mathrm{NO}_{3}{ }^{-}$availability influenced the capability of the plants to manage the content of $\mathrm{NH}_{4}{ }^{+}$in the cells, probably due also to its action as an osmolyte. Moreover, the study contributes to a better understanding of plant $\mathrm{N}$ metabolism, providing novel information about molecular mechanisms involved in plant adaptation to different $\mathrm{N}$ sources.

\section{Results and Discussion}

This study was devised to investigate biochemical responses specific to $\mathrm{NO}_{3}{ }^{-}$or $\mathrm{NH}_{4}{ }^{-}$ availability, as well as the interactions between the two nutrients in co-provision, in maize plants during early vegetative growth. Seedlings were grown in a hydroponic system with low $\mathrm{N}$ availability $\left(1 \mathrm{mM} \mathrm{NO}_{3}{ }^{-}, 125 \mathrm{\mu M} \mathrm{NH}_{4}{ }^{+}\right)$for a total of nine days until the expansion of the second leaf, and then exposed to one of three $\mathrm{N}$ treatments: $5 \mathrm{mM} \mathrm{NO}_{3}{ }^{-}$(n); $5 \mathrm{mM} \mathrm{NH}_{4}{ }^{+}$(a); $2.5 \mathrm{mM} \mathrm{NO}_{3}{ }^{-}+2.5 \mathrm{mM} \mathrm{NH}_{4}{ }^{+}$ (na). This experimental design was chosen in order to expose maize seedlings to the same availability of total $\mathrm{N}$, while changing the proportion between $\mathrm{NO}_{3}{ }^{-}$and $\mathrm{NH}_{4}{ }^{+}$. Moreover, since maize is generally fertilized by a single application at sowing [21], this growth stage corresponds to a period in which maize plants are exposed to high levels of $\mathrm{N}$ and in which they often show the highest susceptibility to an excess of $\mathrm{NH}_{4}^{+}$[22]. Plants were analyzed for a period of three days ( $\mathrm{t} 0,6 \mathrm{~h}, 30 \mathrm{~h}$, and $\left.54 \mathrm{~h}\right)$ to appreciate both early biochemical responses and metabolic acclimations in roots and leaves. After the evaluation of plant growth, nutritional parameters were analyzed in combination with proteomic changes in both organs. 


\subsection{Plant Growth and Metabolic Status of Roots and Leaves}

The estimation of plant growth, measured as the biomass of roots and leaves during the different nutritional treatments, revealed that the two organs grew with a different dynamic (Figure 1).
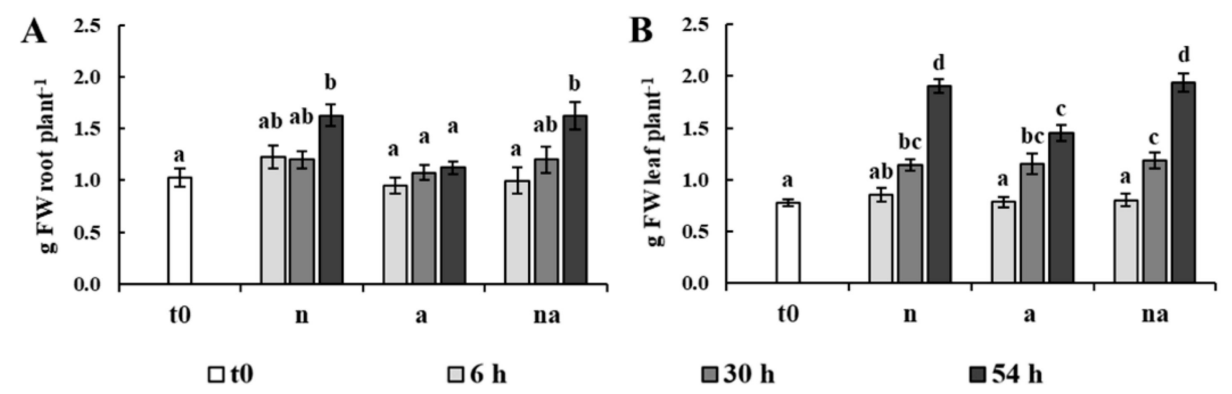

Figure 1. Plant growth evaluated as the fresh biomass of roots (A) and leaves (B) per plant (g FW plant $^{-1}$ ). Maize plants were collected at $\mathrm{t} 0$ (white bar) or after $6 \mathrm{~h}$ (light grey bars), $30 \mathrm{~h}$ (grey bars) and $54 \mathrm{~h}$ (dark grey bars) of growth in the presence of $5 \mathrm{mM} \mathrm{NO}_{3}^{-}$(n), $5 \mathrm{mM} \mathrm{NH}_{4}^{+}$(a) and of $2.5 \mathrm{mM} \mathrm{NO}_{3}{ }^{-}+2.5 \mathrm{mM} \mathrm{NH}_{4}{ }^{+}$(na). Values are the mean \pm standard error (SE) $(n=8)$. The statistical significance was assessed by analysis of variance (ANOVA) test ( $p<0.05$, Tukey post hoc method).

In plants exposed to $\mathrm{NO}_{3}{ }^{-}$and to co-provision (n, na), root growth was appreciable at the third day, resulting in a biomass increment of about $60 \%$ compared with the plants at $t 0$. However, leaf growth was already evident after $30 \mathrm{~h}$ in all conditions, leading to a doubling of the biomass at $54 \mathrm{~h}$ (Figure 1). This behavior is in agreement with the fact that an increase in $\mathrm{N}$ supply promotes an increase in biomass shoot/root ratio in several plant species [3,23]. Although this observation was similar in all conditions, plants supplied only with $\mathrm{NH}_{4}{ }^{+}$(a) were characterized by a much slower increase in the fresh weight of the roots and showed the lowest leaf growth (Figure 1). These results confirm previous studies reporting that $\mathrm{NH}_{4}{ }^{+}$-fed maize plants accumulate less biomass than those fed with $\mathrm{NO}_{3}{ }^{-}$[24]. Plants grown in co-provision (na) showed a biomass accumulation very similar to that of the $\mathrm{NO}_{3}{ }^{-}$-fed plants (n, Figure 1), suggesting that the lower level of $\mathrm{NH}_{4}{ }^{+}$in the growth medium and/or the presence of $\mathrm{NO}_{3}{ }^{-}$led to a reduction of the negative effects caused by a sole $\mathrm{NH}_{4}{ }^{+}$nutrition.

The levels of $\mathrm{NO}_{3}{ }^{-}, \mathrm{NH}_{4}{ }^{+}$, and amino acids were determined in roots and leaves (Figure 2).

In the (n) condition, the content of $\mathrm{NO}_{3}{ }^{-}$reached the highest level at $30 \mathrm{~h}$ in roots and at $54 \mathrm{~h}$ in leaves, which in comparison to the t0 plants corresponded to an increment of about $140 \%$ and $45 \%$, respectively (Figure 2A,B). The plants grown in co-provision (na) showed a similar accumulation of $\mathrm{NO}_{3}{ }^{-}$in both organs, even though the availability of the anion in the growth medium was only half (Figure 2A,B). This observation indicates that the accumulation of the anion was not proportional to the external availability, but it was probably regulated by the requirements of the plants. Moreover, the copresence of $\mathrm{NH}_{4}{ }^{+}$did not seem to modify this process. These results seem to be inconsistent with the observation that $\mathrm{NO}_{3}{ }^{-}$uptake is reduced in presence of $\mathrm{NH}_{4}{ }^{+}$. However, in barley (Hordeum vulgare L.) and in Arabidopsis this effect was ascribed to an inhibition of the inducible high affinity transport systems $[25,26]$, and it is therefore conceivable that it did not influence the accumulation of $\mathrm{NO}_{3}{ }^{-}$in plants exposed to high $\mathrm{N}$ input $(>1 \mathrm{mM})$ for several hours. At the same time, the (a) plants showed a gradual decline in $\mathrm{NO}_{3}{ }^{-}$levels in roots and leaves (Figure $2 \mathrm{~A}, \mathrm{~B}$ ), probably because the plants continued to use the $\mathrm{NO}_{3}{ }^{-}$reserves in the presence of $\mathrm{NH}_{4}{ }^{+}$.

In (n) plants, the content of $\mathrm{NH}_{4}{ }^{+}$did not change in roots, while it showed some fluctuations in leaves (Figure 2C,D). However, in plants exposed to $\mathrm{NH}_{4}{ }^{+}$and to co-provision (a, na) the content of the cation greatly increased, especially in roots, but it never reached levels associated with $\mathrm{NH}_{4}{ }^{+}$ toxicity in maize [22,27]. In these conditions (a, na), in roots the $\mathrm{NH}_{4}{ }^{+}$content already surged up at $6 \mathrm{~h}$, while an increase of foliar $\mathrm{NH}_{4}{ }^{+}$was evident only after $54 \mathrm{~h}$ (Figure 2C,D). 

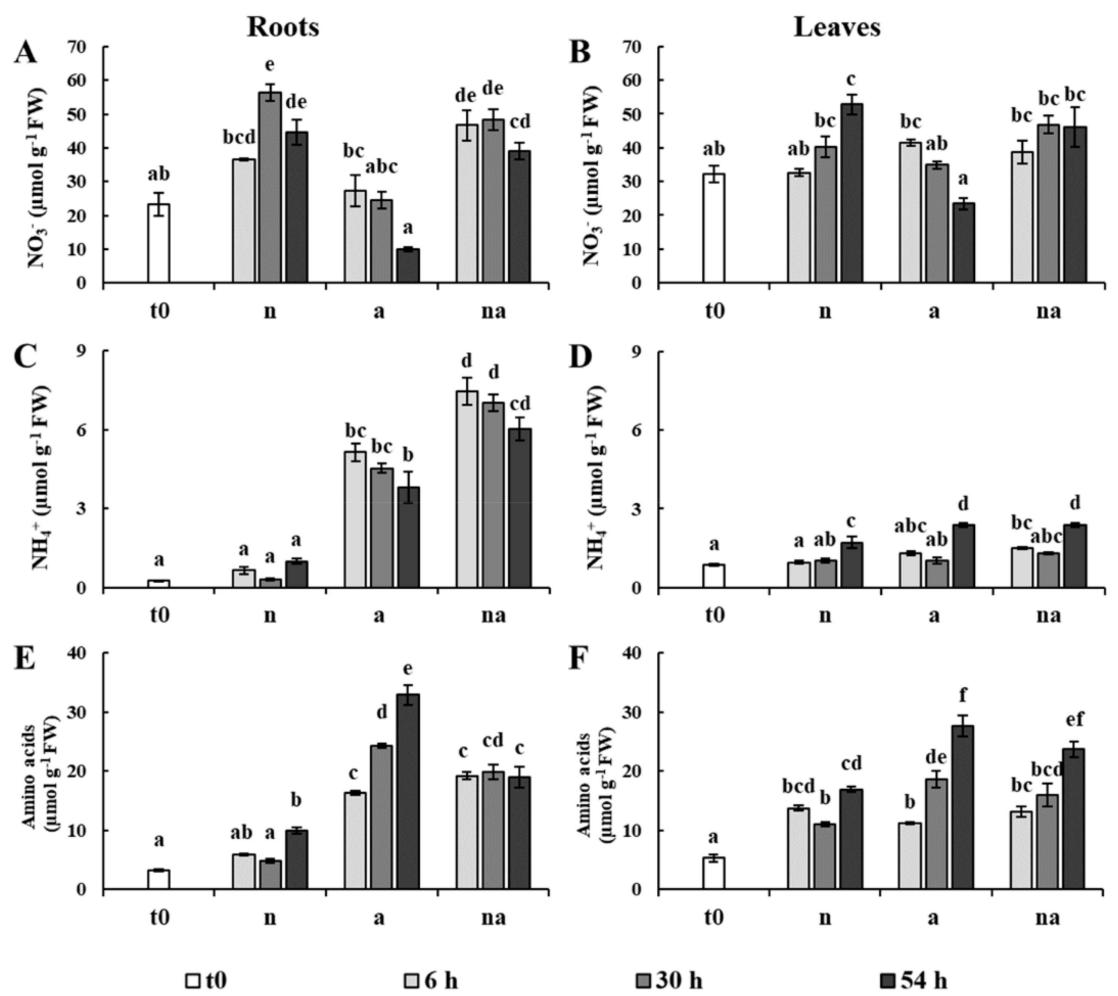

Figure 2. Content of $\mathrm{NO}_{3}{ }^{-}, \mathrm{NH}_{4}{ }^{+}$and amino acids in roots and leaves. The graphs report the content of $\mathrm{NO}_{3}{ }^{-}$in roots $(\mathbf{A})$ and in leaves $(\mathbf{B})$; the content of $\mathrm{NH}_{4}{ }^{+}$in roots $(\mathbf{C})$ and in leaves (D); the content of amino acids in roots (E) and in leaves (F). Maize plants were collected at $\mathrm{t} 0$ (white bar) or after $6 \mathrm{~h}$ (light grey bars), $30 \mathrm{~h}$ (grey bars), and $54 \mathrm{~h}$ (dark grey bars) of growth in presence of $5 \mathrm{mM} \mathrm{NO}_{3}{ }^{-}$ (n), $5 \mathrm{mM} \mathrm{NH}_{4}{ }^{+}(\mathrm{a})$, and $2.5 \mathrm{mM} \mathrm{NO}_{3}{ }^{-}+2.5 \mathrm{mM} \mathrm{NH}_{4}{ }^{+}$(na). Values are the mean $\pm \mathrm{SE}(n=3)$. The statistical significance was assessed by ANOVA test $(p<0.05$, Tukey post hoc method).

In this context, it is important to note that in (a) plants, $54 \mathrm{~h}$ of treatment led to both the highest accumulation of $\mathrm{NH}_{4}{ }^{+}$and to an almost total depletion of $\mathrm{NO}_{3}{ }^{-}$(Figure 2). However, the roots of the plants in co-provision (na) were characterized by an $\mathrm{NH}_{4}{ }^{+}$content much higher than those of the (a) plants, even though the external availability was only half (Figure 2C).

The amino acid levels rose in both organs in all the nutritional conditions. This increase was higher in plants exposed to $\mathrm{NH}_{4}{ }^{+}$and to co-provision (a, na, Figure 2E,F), in agreement with the observation that, in maize, ammonium-fed plants show higher contents of amino-N compounds than nitrate-fed ones [24]. Interestingly, in roots the accumulation of amino acids was not proportional to the content of $\mathrm{NH}_{4}{ }^{+}$. Indeed, the (a) roots were characterized by an upsurge of amino acid levels at $54 \mathrm{~h}$, which was not evident in (na) plants (Figure 2E). These results suggest that the availability of $\mathrm{NO}_{3}{ }^{-}$could have exerted positive effects on the storage capacity of $\mathrm{NH}_{4}{ }^{+}$of the plants.

To investigate this hypothesis, we calculated the total content of $\mathrm{N}$ in plants as well as the ratio between the inorganic $\mathrm{N}$ and organic $\mathrm{N}$ in roots and leaves (Table 1). The total $\mathrm{N}$ content in plants at $54 \mathrm{~h}$ reached similar values in all the nutritional treatments. However, plants showed very different partitioning between inorganic and organic forms of $\mathrm{N}$. The (a) plants were characterized by a particular decrease in this ratio, due to the highest increase in amino acid and protein levels. On the contrary, plants in co-provision showed values more similar to (n) plants, although they had the highest $\mathrm{NH}_{4}{ }^{+}$content in roots (Figure 2). These results indicate that in co-provision the presence of $\mathrm{NO}_{3}{ }^{-}$promotes a major capability of storage of $\mathrm{NH}_{4}{ }^{+}$in the roots, and therefore it could contribute to alleviating metabolic stress by reducing the need to assimilate the cation into amino acids. 
Table 1. Total nitrogen $(\mathrm{N})$ in plants and the ratio between the inorganic and organic forms in roots and leaves. Maize plants were collected at t0 and after $6 \mathrm{~h}, 30 \mathrm{~h}$, and $54 \mathrm{~h}$ of growth in presence of $5 \mathrm{mM} \mathrm{NO}_{3}{ }^{-}$(n), $5 \mathrm{mM} \mathrm{NH}_{4}^{+}$(a), and $2.5 \mathrm{mM} \mathrm{NO}_{3}{ }^{-}+2.5 \mathrm{mM} \mathrm{NH}_{4}{ }^{+}$(na). Values are the mean $\pm \mathrm{SE}$ $(n=3)$. The statistical significance was assessed by ANOVA test $(p<0.05$, Tukey post hoc method).

\begin{tabular}{|c|c|c|c|c|}
\hline Condition & Time (h) & $\begin{array}{l}\text { Total N Plant } \\
\left(\mathrm{mg} \mathrm{g}^{-1} \text { FW) }\right.\end{array}$ & $\begin{array}{c}\text { Inorganic N/Organic N } \\
\text { Roots }\end{array}$ & $\begin{array}{c}\text { Inorganic N/Organic N } \\
\text { Leaves }\end{array}$ \\
\hline & 0 & $3.37 \pm 0.02(\mathrm{a})$ & $0.50 \pm 0.07(\mathrm{bc})$ & $0.24 \pm 0.03(\mathrm{abc})$ \\
\hline \multirow{3}{*}{$\begin{array}{l}\text { Nitrate } \\
\text { (n) }\end{array}$} & 6 & $3.73 \pm 0.06(\mathrm{ab})$ & $0.80 \pm 0.01(\mathrm{de})$ & $0.23 \pm 0.01(a b)$ \\
\hline & 30 & $4.25 \pm 0.10(\mathrm{~cd})$ & $1.28 \pm 0.10(\mathrm{f})$ & $0.26 \pm 0.02(\mathrm{bc})$ \\
\hline & 54 & $4.38 \pm 0.10(\mathrm{~cd})$ & $0.91 \pm 0.07(\mathrm{e})$ & $0.34 \pm 0.01(\mathrm{c})$ \\
\hline \multirow{3}{*}{$\begin{array}{l}\text { Ammonium } \\
\text { (a) }\end{array}$} & 6 & $4.13 \pm 0.04(\mathrm{bc})$ & $0.49 \pm 0.08(\mathrm{bc})$ & $0.28 \pm 0.01(\mathrm{bc})$ \\
\hline & 30 & $4.55 \pm 0.03(\mathrm{de})$ & $0.34 \pm 0.03(\mathrm{ab})$ & $0.21 \pm 0.01(\mathrm{ab})$ \\
\hline & 54 & $4.50 \pm 0.06(\mathrm{cde})$ & $0.13 \pm 0.01(\mathrm{a})$ & $0.14 \pm 0.01(\mathrm{a})$ \\
\hline Nitrate + & 6 & $4.34 \pm 0.14(\mathrm{~cd})$ & $0.74 \pm 0.02(\mathrm{cde})$ & $0.28 \pm 0.03(\mathrm{bc})$ \\
\hline Ammonium & 30 & $4.90 \pm 0.10(\mathrm{e})$ & $0.74 \pm 0.07(\mathrm{cde})$ & $0.28 \pm 0.02(\mathrm{bc})$ \\
\hline (na) & 54 & $4.60 \pm 0.06(\mathrm{de})$ & $0.61 \pm 0.04(\mathrm{bcd})$ & $0.30 \pm 0.04(\mathrm{bc})$ \\
\hline
\end{tabular}

The evaluation of the contents of sucrose and reducing sugars showed that, after a little increase at $6 \mathrm{~h}$, the levels were only slightly affected by the nutritional treatments, except for a remarkable doubling in the content of reducing sugars in the roots of the (a) plants, not found in co-provision (Figure 3). Although this had already been observed in another maize genotype [27], further studies are needed to clarify the metabolic meaning of this accumulation. However, this result weakens the possibility that the lack of root growth of the (a) plants was due to an insufficient allocation of photoassimilates, as previously proposed in other plant species [2,10].
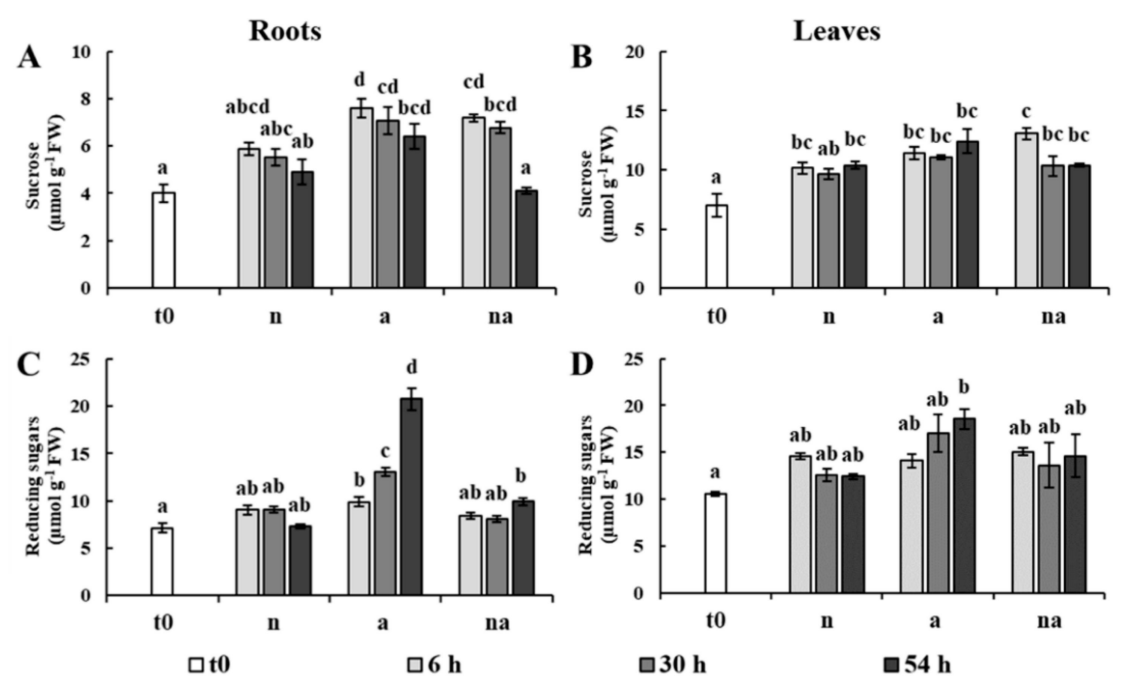

Figure 3. Contents of sucrose $(\mathbf{A}, \mathbf{B})$ and reducing sugars $(\mathbf{C}, \mathbf{D})$ in roots $(\mathbf{A}, \mathbf{C})$ and leaves $(\mathbf{B}, \mathbf{D})$. Maize plants were collected at $\mathrm{t} 0$ (white bar) or after $6 \mathrm{~h}$ (light grey bars), $30 \mathrm{~h}$ (grey bars), or $54 \mathrm{~h}$ (dark grey bars) of growth in presence of $5 \mathrm{mM} \mathrm{NO}_{3}{ }^{-}$(n), $5 \mathrm{mM} \mathrm{NH}_{4}{ }^{+}$(a) and $2.5 \mathrm{mM} \mathrm{NO}_{3}{ }^{-}+2.5 \mathrm{mM} \mathrm{NH}_{4}{ }^{+}$(na). Values are the mean \pm SE $(n=3)$. The statistical significance was assessed by ANOVA test $(p<0.05$, Tukey post hoc method).

\subsection{Comparative Proteomic Analyses in Roots and Leaves of Maize during Exposure to Different N Sources}

The analysis of the proteomic changes in roots and leaves of maize plants exposed to different $\mathrm{N}$ sources ( $\mathrm{n}$, a and na) was done by comparing the total proteome of each organ among all the conditions (three nutritional treatments for three timings). The comparison was performed by means of gel liquid chromatography-mass spectrometry (GeLC-MS/MS): proteins are purified by sodium 
dodecyl sulphate-polyacrylamide gel electrophoresis (SDS-PAGE), in-gel digested, and then identified and quantified by mass spectrometry [28]. This approach allowed us to analyze the abundance of 336 and 246 proteins in roots and leaves, respectively, with high reliability in identification and a good degree of comparability among samples and conditions (Table 2, Supplementary Data 1).

Table 2. Evaluation of the comparative proteomic analyses in roots and leaves of maize.

\begin{tabular}{lcc}
\hline \multicolumn{1}{c}{ Parameter } & Root & Leaf \\
\hline$n$. of total peptides & 39,516 & 31,739 \\
Average of peptides per sample $( \pm \mathrm{SE}, n=27)$ & $1464 \pm 15$ & $1176 \pm 11$ \\
Average of peptides per condition $( \pm \mathrm{SE}, n=9)$ & $4391 \pm 55$ & $3527 \pm 50$ \\
Average of unique peptide per protein $( \pm \mathrm{SE})$ & $6.8 \pm 0.3$ & $6.4 \pm 0.4$ \\
Average amino acid coverage $\%( \pm \mathrm{SE})$ & $22.5 \pm 0.8$ & $22.4 \pm 0.9$ \\
$n$. of identified proteins & 336 & 246 \\
$n$. of differentially accumulated proteins $(\%)$ & $51(15 \%)$ & $48(19 \%)$ \\
\hline
\end{tabular}

The proteins that showed changes in abundance of at least two-fold in at least two conditions were further selected by means of the two-way ANOVA to find the main source of variation (time, $\mathrm{N}$ sources), and by means of the Tukey-test $(p<0.05)$ to evaluate the differences among conditions. The selected proteins were named Differentially abundant Proteins (DPs). The DPs identification in roots and leaves, their changes and statistics, are reported in Tables 3 and 4, respectively.

The cluster representations of the DP abundances in roots and leaves are shown in Figures 4 and 5 (for detailed bar graphs reporting protein levels see Supplementary Data 2).

The DPs accounted for $15 \%$ and $19 \%$ of the quantified proteins in roots and leaves, respectively (Table 2), showing that the two organs were affected by the treatments to a similar extent. This result is in agreement with our previous two-dimensional gel electrophoresis (2-DE) study, showing comparable effects on root and leaf proteome in maize plants exposed to $\mathrm{NO}_{3}{ }^{-}$[20]. In contrast, a microarray study in Arabidopsis indicated that the responses to $\mathrm{NO}_{3}{ }^{-}$were much more ample in roots than in shoots [29], but this discrepancy probably derives from different approaches and/or nutritional treatments.

The functional classification of the DPs recognized nine main classes, partially overlapping in roots and leaves (Figure 6). "Protein synthesis and folding" was the main category in both organs, indicating a general reprogramming of plant functionalities. Leaves were also characterized by changes in DPs involved in photosynthesis $(15 \%)$ and energy metabolism $(10 \%)$, while in roots the DPs related to C metabolism (mainly catabolic processes) were predominant (18\%). Moreover, the root proteomic profile was characterized by changes in proteins involved in cell water homeostasis $(8 \%)$ and cell wall ( $4 \%)$.

The classification of DPs according to the main source of variation (Figure 7, Tables 3 and 4, Supplementary Data 1) discriminated between the DPs specifically affected by the N source and the DPs that were not influenced by this factor (changes in which were related to time and/or to total $\mathrm{N}$ availability). In roots, most of the DPs were specifically affected by the $\mathrm{N}$ source (Figure 7A), highlighting that $\mathrm{NO}_{3}{ }^{-}$and $\mathrm{NH}_{4}{ }^{+}$led to distinct effects. This result is in agreement with a study showing that in Arabidopsis plants exposed for $1.5 \mathrm{~h}$ to $\mathrm{NO}_{3}{ }^{-}$or $\mathrm{NH}_{4}{ }^{+}$more than $40 \%$ of the transcriptomic changes in roots were nitrate- or ammonium-specific [30]. However, in the leaf proteome the DPs were more equally distributed between the two categories (Figure 7B), indicating that the leaf metabolism was less specifically affected by the kind of $\mathrm{N}$ source respect than the root one. Overall, this response was consistent with the physiological and biochemical data. Indeed, the treatments differently affected root growth but all of them sustained an increment in leaf biomass. Moreover, the nutritional treatments induced metabolic changes which were more different among conditions in roots than in leaves (Figures 1-3). As highlighted by previous studies [20,22,27], this proteomic profiling confirmed the fundamental role of roots in plant adaptation to the kind of $\mathrm{N}$ source. 
Table 3. Proteins differentially accumulated in root proteome. Proteins are grouped according to the functional classifications. FC: maximum fold change among conditions, n.d.: not detectable, the protein was absent in at least one condition. V: main source of variation (two-way ANOVA, $p<0.05$ ); t: time, s: N source; i: interaction. Differences: h: hours of exposure to $5 \mathrm{mM} \mathrm{NO}_{3}^{-}$(n), $5 \mathrm{mM} \mathrm{NH}_{4}{ }^{+}$(a), and $2.5 \mathrm{mM} \mathrm{NO}_{3}{ }^{-}+2.5 \mathrm{mM} \mathrm{NH}_{4}{ }^{+}$(na). ${ }^{\text {a}}$ : annotated by basic local alignment search tool (BLAST). Different letters indicate significant difference $(* p<0.05$, Tukey post-hoc); letters are arranged in ascending order according to the increase in protein abundance. Bold letters indicate significant difference within each N source. NADH: Nicotinamide adenine dinucleotide; NADP: Nicotinamide adenine dinucleotide phosphate; PIP: plasma membrane intrinsic proteins; TIP: tonoplast intrinsic proteins.

\begin{tabular}{|c|c|c|c|c|c|c|c|c|c|c|c|c|c|c|}
\hline \multirow{3}{*}{ ID } & \multirow{3}{*}{ Entry-Protein Name } & \multirow{3}{*}{ FC } & & & & \multicolumn{9}{|c|}{ Differences } \\
\hline & & & \multicolumn{3}{|c|}{$\mathrm{v}$} & \multicolumn{3}{|c|}{$h(n)$} & \multicolumn{3}{|c|}{ h (a) } & \multicolumn{3}{|c|}{ h (na) } \\
\hline & & & $t$ & $\mathrm{~s}$ & i & 06 & 30 & 54 & 06 & 30 & 54 & 06 & 30 & 54 \\
\hline \multicolumn{15}{|c|}{ Nitrogen assimilation } \\
\hline R74 & B6SY01-Ferredoxin-nitrite reductase & 7.57 & & & * & abc & d & $\mathrm{cd}$ & $a b c$ & $\mathrm{ab}$ & a & $a b c$ & bcd & $\mathrm{abc}$ \\
\hline R64 & B4FR61—Glutamine synthetase (cytosol) & 2.60 & & & * & a & a & a & ab & c & c & a & a & cb \\
\hline R189 & A0A1D6NFK0 - Glutamate synthase 1 [NADH] chloroplastic & 8.54 & * & * & & $\mathrm{a}$ & $\mathrm{ab}$ & a & a & $a b$ & b & a & a & $a b$ \\
\hline R103 & B5U8J8-Asparagine synthetase & n.d. & & & * & a & $\mathrm{a}$ & a & a & $\mathbf{b}$ & c & a & a & a \\
\hline R68 & B4G043-Ferredoxin-NADP reductase & 5.48 & & & * & $\mathrm{cb}$ & $\mathrm{cb}$ & $\mathrm{c}$ & cb & $a b$ & a & $\mathrm{cb}$ & $\mathrm{ab}$ & $\mathrm{abc}$ \\
\hline \multicolumn{15}{|c|}{ Amino acid metabolism } \\
\hline R6 & B6U4D6-Phenylalanine ammonia-lyase & 2.88 & & & * & $\mathrm{bc}$ & $a b c$ & $\mathrm{bc}$ & bc & $\mathbf{a}$ & a & c & abc & ab \\
\hline R107 & P80608-Cysteine synthase & 2.74 & & & * & $\mathbf{a}$ & bc & ab & $a b$ & $a b$ & c & a & bc & $\mathrm{abc}$ \\
\hline R49 & A0A1D6FUX8 - S-adenosylmethionine synthase & 2.16 & * & & & $\mathbf{b}$ & $\mathbf{a}$ & $a b$ & $a b$ & $\mathrm{ab}$ & $a b$ & $\mathrm{~b}$ & $\mathrm{ab}$ & $\mathrm{ab}$ \\
\hline R96 & K7TSD2-Serine hydroxymethyltransferase & 2.08 & * & & & $\mathrm{b}$ & $a b$ & $a b$ & $a b$ & $\mathrm{ab}$ & a & $a b$ & a & a \\
\hline \multicolumn{15}{|c|}{ Carbon metabolism } \\
\hline R207 & C0PMR3-Glucose-6-phosphate 1-dehydrogenase (cytosol) & 7.66 & * & & & $\mathrm{ab}$ & $\mathrm{b}$ & $\mathrm{ab}$ & $\mathrm{a}$ & $\mathrm{ab}$ & $\mathrm{ab}$ & $\mathrm{ab}$ & $\mathrm{ab}$ & $\mathrm{ab}$ \\
\hline R85 & A0A1D6J424-Glucose-6-phosphate 1-dehydrogenase (chloroplast) & 224 & & * & & $a b$ & $\mathrm{~b}$ & $\mathrm{ab}$ & $\mathrm{ab}$ & $\mathrm{a}$ & a & $\mathrm{ab}$ & $\mathrm{ab}$ & $\mathrm{ab}$ \\
\hline R57 & A0A1D6NVZ7-Transketolase 1 & 2.98 & * & * & & $a b$ & a & $\mathrm{ab}$ & $\mathrm{ab}$ & a & a & b & $a b$ & $\mathbf{a}$ \\
\hline R231 & Q8L5G8-Phosphotransferase & 7.59 & * & & & $a b$ & $\mathrm{~b}$ & $\mathrm{ab}$ & a & $\mathrm{ab}$ & $a b$ & a & $a b$ & $\mathrm{ab}$ \\
\hline R168 & A0A1D6NR86-Pyrophosphate-fructose 6-phosphate 1-phosphotransferase subunit beta & 2.85 & & & * & a & b & ab & a & a & a & a & $\mathbf{b}$ & $\mathrm{ab}$ \\
\hline $\mathrm{R} 243$ & B4F820-Triosephosphate isomerase ${ }^{\mathbf{a}}$ & 3.67 & * & & & a & $\mathrm{ab}$ & $a b c$ & a & $a b$ & c & $\mathrm{abc}$ & $a b c$ & $\mathrm{bc}$ \\
\hline R19 & C0HHU2-2,3-bisphosphoglycerate-independent phosphoglycerate mutase 1 & 2.35 & & & * & a & $\mathrm{ab}$ & $\mathrm{ab}$ & a & $a b$ & c & a & a & cb \\
\hline R119 & A0A1D6H4C4-Citrate synthase & 2.87 & & & * & $a b$ & $\mathrm{ab}$ & a & a & $\mathbf{a b}$ & b & a & $\mathrm{ab}$ & a \\
\hline $\mathrm{R} 28$ & Q9SAZ6-Phosphoenolpyruvate carboxylase & 3.20 & * & * & & a & $a b$ & a & $a b$ & $\mathrm{ab}$ & $\mathrm{b}$ & a & $\mathrm{ab}$ & $a b$ \\
\hline \multicolumn{15}{|c|}{ Cell water homeostasis } \\
\hline $\mathrm{R} 290$ & B6T7A1-Voltage-gated potassium channel beta subunit & 2.21 & * & * & & $a b$ & c & bc & $\mathrm{ab}$ & $\mathrm{ab}$ & $\mathrm{ab}$ & $\mathrm{a}$ & $a b c$ & $\mathrm{abc}$ \\
\hline R127 & B6T634-Aquaporin PIP2.1 & 2.75 & & & * & $a b$ & $a b$ & $\mathrm{~b}$ & $\mathrm{ab}$ & $\mathrm{ab}$ & a & $\mathrm{ab}$ & $\mathrm{b}$ & $\mathrm{b}$ \\
\hline R193 & A0A1R3N4Y1-Aquaporin PIP2-5 & 5.52 & * & * & & $\mathbf{a}$ & $a b c$ & c & $\mathrm{ab}$ & $a b c$ & $a b$ & $a b c$ & $a b c$ & $\mathrm{bc}$ \\
\hline $\mathrm{R} 285$ & B6TNY0-Aquaporin TIP2.1 & 2.81 & * & * & & $\mathrm{ab}$ & $\mathrm{ab}$ & $\mathrm{b}$ & $\mathrm{ab}$ & $\mathrm{a}$ & $a b$ & $\mathrm{ab}$ & $\mathrm{ab}$ & $\mathrm{ab}$ \\
\hline \multicolumn{15}{|c|}{ Cell wall metabolism } \\
\hline R92 & A0A1D6IMH7-Hydroxyproline-rich glycoprotein family protein & 4.07 & & & * & b & $\mathbf{a}$ & $\mathbf{a}$ & $\mathrm{ab}$ & $\mathrm{ab}$ & $\mathrm{ab}$ & $\mathrm{ab}$ & $\mathrm{a}$ & $\mathrm{ab}$ \\
\hline R154 & B6UD26-O-methyltransferase ZRP4 & 3.66 & & & * & $a b$ & $a b$ & $\mathrm{ab}$ & a & a & b & $\mathrm{ab}$ & $\mathrm{ab}$ & $\mathrm{ab}$ \\
\hline
\end{tabular}


Table 3. Cont.

\begin{tabular}{|c|c|c|c|c|c|c|c|c|c|c|c|c|c|c|}
\hline \multirow{3}{*}{ ID } & \multirow{3}{*}{ Entry—Protein Name } & \multirow{3}{*}{ FC } & & & & \multicolumn{9}{|c|}{ Differences } \\
\hline & & & \multicolumn{3}{|c|}{$\mathrm{v}$} & \multicolumn{3}{|c|}{$h(n)$} & \multicolumn{3}{|c|}{ h (a) } & \multicolumn{3}{|c|}{$\mathrm{h}(\mathrm{na})$} \\
\hline & & & $\mathbf{t}$ & $\mathrm{s}$ & $\mathrm{i}$ & 06 & 30 & 54 & 06 & 30 & 54 & 06 & 30 & 54 \\
\hline \multicolumn{15}{|c|}{ Protein synthesis and folding } \\
\hline R58 & C0PCQ6-40S ribosomal protein S2-1 & 3.21 & * & & & c & a & ab & $\mathrm{bc}$ & $a b c$ & $a b c$ & $\mathrm{bc}$ & $\mathrm{ab}$ & $a b c$ \\
\hline R255 & A0A1D6PYT7-Ribosomal protein S25 & 2.80 & & & * & $\mathrm{bc}$ & $\mathrm{ab}$ & $\mathrm{ab}$ & c & $a b c$ & $\mathbf{a}$ & $a b c$ & $\mathrm{ab}$ & $a b$ \\
\hline R309 & A0A1D6FKZ4-60S acidic ribosomal protein P2-5 & 6.66 & * & & & $\mathbf{a}$ & b & ab & $a b$ & $\mathrm{ab}$ & a & $a b$ & $a b$ & $\mathrm{ab}$ \\
\hline R87 & C4JA45-60S ribosomal protein L5-1 homolog b & 2.52 & * & & & $\mathbf{a}$ & b & $\mathbf{b}$ & $\mathrm{ab}$ & $\mathrm{ab}$ & $\mathrm{b}$ & $a b$ & $\mathrm{~b}$ & $a b$ \\
\hline R122 & B6T267-Ribosomal protein L15 & 18.7 & * & & & $\mathrm{bc}$ & $\mathrm{ab}$ & $a b c$ & c & $a b c$ & $a b c$ & $a b c$ & a & $a b c$ \\
\hline R4 & B7ZZ42-Heat shock $70 \mathrm{kDa}$ protein 3 & 2.25 & & & * & bc & a & $a b c$ & $\mathrm{bc}$ & $a b c$ & $\mathrm{ab}$ & c & a & $a b$ \\
\hline R100 & B6U0V6-Endoplasmin & 5.19 & * & & & $\mathrm{ab}$ & $\mathrm{ab}$ & $\mathrm{ab}$ & a & $\mathrm{ab}$ & a & $a b$ & $\mathrm{~b}$ & $\mathrm{ab}$ \\
\hline R188 & B6SXW8-RuBisCO large subunit-binding protein sub. alpha & 3.92 & * & & & a & $\mathrm{ab}$ & $\mathrm{ab}$ & a & $\mathrm{ab}$ & a & a & b & $a b$ \\
\hline R297 & A0A1D6FAH0-T-complex protein 1 subunit epsilon & 4.66 & * & & & $\mathrm{ab}$ & $\mathrm{ab}$ & a & $\mathrm{ab}$ & $\mathrm{ab}$ & a & $\mathrm{ab}$ & $\mathrm{b}$ & $\mathrm{ab}$ \\
\hline R131 & B4FZZ2-Peptidyl-prolyl cis-trans isomerase & 4.68 & * & & & c & a & ab & c & $\mathbf{a}$ & $a b$ & bc & $\mathbf{a}$ & $a b$ \\
\hline R216 & A0A1D6LN79-Peptidyl-prolyl cis-trans isomerase & 15.7 & * & & & d & $\mathbf{a}$ & $a b c$ & $\mathrm{~cd}$ & abcd & abcd & bcd & $\mathrm{ab}$ & $\mathrm{abcd}$ \\
\hline R145 & A0A1D6F8L7—Coatomer subunit gamma & 3.43 & * & & & a & $\mathrm{ab}$ & $\mathrm{ab}$ & a & a & $\mathrm{a}$ & a & $\mathbf{b}$ & $\mathbf{a}$ \\
\hline R205 & A0A1D6PJW1-Proteasome subunit alpha type & n.d. & * & * & & a & a & a & b & a & a & a & $\mathrm{a}$ & a \\
\hline R230 & B4FB55-Ras-related protein ARA-3 & 2.40 & * & * & & $a b$ & $\mathrm{~b}$ & $\mathrm{~b}$ & a & $\mathrm{ab}$ & $\mathrm{ab}$ & $a b$ & $\mathrm{~b}$ & $\mathrm{ab}$ \\
\hline \multicolumn{15}{|c|}{ Stress response and other functions } \\
\hline R105 & C0P848-Formate dehydrogenase, mitochondrial & 14.2 & * & & & $\mathrm{a}$ & $\mathrm{a}$ & $\mathrm{a}$ & a & a & b & $\mathrm{a}$ & $\mathrm{ab}$ & $\mathrm{ab}$ \\
\hline R99 & B8A2B4-Guanine nucleotide-binding protein beta subunit-like protein a & 2.22 & * & & & abc & a & c & $a b$ & a & bc & $\mathrm{bc}$ & $\mathrm{ab}$ & $\mathrm{bc}$ \\
\hline R112 & A5H452-Peroxidase 70 & 2.75 & * & & & c & a & $a b c$ & $a b c$ & $a b c$ & $a b c$ & $\mathrm{bc}$ & $a b$ & $\mathrm{ab}$ \\
\hline R133 & A0A1D6E530-Peroxidase & 3.56 & * & & & $\mathrm{ab}$ & $\mathrm{b}$ & $\mathrm{ab}$ & $a b$ & $\mathrm{ab}$ & $\mathrm{ab}$ & a & $a b$ & $a b$ \\
\hline R300 & B6SIU4-Peroxidase & n.d. & & & * & bc & $a b$ & c & $a b c$ & $\mathrm{ab}$ & a & abc & $a b c$ & $a b$ \\
\hline R31 & C0PH85-Tubulin beta chain & 2.88 & * & * & & d & cb & cb & c & $a b$ & a & c & $\mathrm{bc}$ & $a b c$ \\
\hline R79 & Q6RW10-Allene oxide synthase & 2.27 & & & * & $\mathbf{a}$ & b & $a b$ & a & $\mathrm{ab}$ & $\mathrm{ab}$ & $a b$ & $\mathrm{ab}$ & $a b$ \\
\hline R225 & A0A1D6HSR3—Putative carboxylesterase 15 & n.d. & & & * & a & a & a & a & $\mathbf{b}$ & c & $\mathbf{a}$ & $a b$ & $\mathbf{b}$ \\
\hline R236 & A0A1D6QNT6-Peroxisomal fatty acid beta-oxidation multifunctional protein a & 3.86 & * & & & a & $\mathrm{ab}$ & $\mathrm{ab}$ & $\mathrm{ab}$ & $\mathrm{ab}$ & $\mathrm{ab}$ & a & b & ab \\
\hline R271 & Q71RX2-Isopentenyl pyrophosphate isomerase & n.d. & * & * & & ab & c & bc & $\mathrm{a}$ & $\mathrm{abc}$ & $\mathrm{abc}$ & a & bc & bc \\
\hline R272 & B6TKK2-Gibberellin receptor GID1L2 & n.d. & * & & & $\mathrm{ab}$ & $\mathrm{ab}$ & $\mathrm{b}$ & $\mathrm{ab}$ & $\mathrm{ab}$ & $\mathrm{ab}$ & a & a & $a b$ \\
\hline R301 & B6TP36-Prohibitin & 3.56 & * & & & $\mathrm{ab}$ & $\mathrm{bc}$ & $a b c$ & $\mathrm{a}$ & $a b c$ & $a b c$ & $a b$ & c & abc \\
\hline R318 & B6T7H0—Glutathione S-transferase 6 & n.d. & & & * & a & ab & b & a & a & a & $\mathrm{a}$ & $\mathrm{a}$ & a \\
\hline
\end{tabular}


Table 4. Proteins differentially accumulated in leaf proteome. Proteins are grouped according to the functional classifications. FC: maximum fold change among conditions, n.d.: not detectable, the protein was absent in at least one condition. V: main source of variation (two-way ANOVA, $p<0.05$ ); t: time, s: N source; i: interaction. Differences: h: hours of exposure to $5 \mathrm{mM} \mathrm{NO}_{3}{ }^{-}$(n), $5 \mathrm{mM} \mathrm{NH}_{4}{ }^{+}$(a), and $2.5 \mathrm{mM} \mathrm{NO}_{3}{ }^{-}+2.5 \mathrm{mM} \mathrm{NH}_{4}{ }^{+}$(na). ${ }^{\text {a: }}$ annotated by BLAST. Different letters indicate significant difference ${ }^{*} p<0.05$, Tukey post-hoc); letters are arranged in ascending order according to the increase in protein abundance. Bold letters indicate significant difference within each N source. ATP: adenosine triphosphate; HSP: heat shock protein.

\begin{tabular}{|c|c|c|c|c|c|c|c|c|c|c|c|c|c|c|}
\hline \multirow{3}{*}{ ID } & \multirow{3}{*}{ Entry-Protein Name } & \multirow{3}{*}{ FC } & & & & \multicolumn{9}{|c|}{ Differences } \\
\hline & & & \multicolumn{3}{|c|}{$\mathrm{V}$} & \multicolumn{3}{|c|}{$h(n)$} & \multicolumn{3}{|c|}{ h (a) } & \multicolumn{3}{|c|}{ h (na) } \\
\hline & & & $t$ & s & $\mathrm{i}$ & 06 & 30 & 54 & 06 & 30 & 54 & 06 & 30 & 54 \\
\hline \multicolumn{15}{|c|}{ Nitrogen assimilation } \\
\hline L149 & B6SY01-Ferredoxin-nitrite reductase & 3.00 & * & & & a & ab & b & a & a & $a b$ & $a b$ & a & $\mathrm{ab}$ \\
\hline L177 & B6TE43-Glutamine synthetase (chloroplast) & 3.92 & * & & & $a b$ & $a b$ & $a b$ & $a b$ & a & $\mathrm{a}$ & b & ab & $\mathbf{a}$ \\
\hline \multicolumn{15}{|c|}{ Amino acid metabolism } \\
\hline L193 & B6TQ06-Aminomethyltransferase & 2.31 & * & & & $a b$ & $a b$ & $\mathrm{~b}$ & $a b$ & a & $a b$ & $a b$ & $a b$ & $\mathrm{ab}$ \\
\hline L22 & C0P5Y3-5-methyltetrahydropteroyltriglutamate -homocysteine methyltransferase 1 (MetE) & 3.57 & * & & & $a b$ & a & $a b$ & $a b$ & $\mathrm{ab}$ & $\mathrm{b}$ & $a b$ & $a b$ & $\mathrm{ab}$ \\
\hline L194 & A0A1D6KDZ0 - Shikimate kinase family protein & 2.85 & * & & & abc & ab & c & $a b$ & ab & c & abc & a & bc \\
\hline \multicolumn{15}{|c|}{ Photosynthesis } \\
\hline L31 & P04966-Photosystem I P700 chlorophyll a apoprotein A1 & 2.48 & * & & & a & ab & b & a & b & ab & $a b$ & $\mathrm{~b}$ & $\mathrm{ab}$ \\
\hline L85 & P04967-Photosystem I P700 chlorophyll a apoprotein A2 & 2.62 & & & * & a & bc & $a b$ & $a b$ & c & $\mathbf{a b}$ & $a b$ & $a b$ & $a b$ \\
\hline L132 & B6U534—Photosystem I reaction center subunit V & 3.85 & * & & & c & c & a & bc & c & a & $\mathrm{bc}$ & $\mathrm{bc}$ & $a b$ \\
\hline L196 & B4FLT7—Photosystem I H subunit1 & 2.99 & * & * & & bcd & d & $a b$ & abc & $\mathrm{cd}$ & a & $a b$ & bcd & a \\
\hline L113 & B6SP64-Oxygen evolving enhancer protein 3 containing protein & 2.34 & * & & & a & a & b & a & a & b & a & $\mathbf{a}$ & $\mathbf{b}$ \\
\hline L162 & B8A3D1-Pyruvate phosphate dikinase regulatory protein, chloroplastic a & 6.68 & * & & & a & a & $a b$ & a & a & b & a & a & $\mathrm{ab}$ \\
\hline L212 & B6TKB3-Triose phosphate/phosphate translocator, non-green plastid, chloroplast & n.d. & * & & & a & a & $a b$ & a & a & b & a & a & $\mathbf{b}$ \\
\hline \multicolumn{15}{|c|}{ Energy metabolism } \\
\hline L115 & B6T908-ATP synthase B chain & 8.19 & * & * & & $a b$ & abc & c & a & $a b$ & $a b c$ & $a b$ & $a b c$ & $\mathrm{bc}$ \\
\hline L225 & B6SP77-ATP synthase $C$ chain & 77.8 & * & & & a & a & b & a & $\mathrm{a}$ & $a b$ & $\mathrm{a}$ & $\mathrm{a}$ & $a b$ \\
\hline L155 & B6T168-ATP synthase epsilon chain & 3.09 & * & & & a & $a b c$ & bc & a & abc & bc & abc & ab & c \\
\hline L130 & B4FK49-Nucleoside diphosphate kinase & 4.88 & * & * & & a & $a b$ & $a b c$ & $a b c$ & $a b c$ & bc & abc & ab & c \\
\hline L236 & C0HHC4-Nucleoside diphosphate kinase & 7.24 & * & & & a & a & b & a & a & b & a & a & b \\
\hline \multicolumn{15}{|c|}{ Carbon metabolism } \\
\hline L192 & A0A1D6N8I0-2,3-bisphosphoglycerate-independent phosphoglycerate mutase 1 & 4.77 & * & & & $a b$ & a & $a b$ & $a b$ & $a b$ & $a b$ & $a b$ & $a b$ & $\mathrm{~b}$ \\
\hline L191 & B8A0W7-Enolase 1 a & 13.1 & * & & & a & a & abc & $a b$ & a & bc & a & a & c \\
\hline L110 & B4FZU8-Malate dehydrogenase & 2.31 & * & & & a & a & b & a & a & b & a & a & b \\
\hline
\end{tabular}


Table 4. Cont

\begin{tabular}{|c|c|c|c|c|c|c|c|c|c|c|c|c|c|c|}
\hline \multirow{3}{*}{ ID } & \multirow{3}{*}{ Entry-Protein Name } & \multirow{3}{*}{ FC } & & & & \multicolumn{9}{|c|}{ Differences } \\
\hline & & & \multicolumn{3}{|c|}{$\mathrm{V}$} & \multicolumn{3}{|c|}{$h(n)$} & \multicolumn{3}{|c|}{ h (a) } & \multicolumn{3}{|c|}{ h (na) } \\
\hline & & & $\mathbf{t}$ & $\mathbf{s}$ & $\mathbf{i}$ & 06 & 30 & 54 & 06 & 30 & 54 & 06 & 30 & 54 \\
\hline \multicolumn{15}{|c|}{ Protein synthesis and folding } \\
\hline L69 & A0A0B4J303-Eukaryotic initiation factor4a & 3.01 & * & & & $\mathrm{abc}$ & a & $\mathrm{abc}$ & $a b$ & $a b$ & bc & $a b$ & a & c \\
\hline L111 & A0A1D6LAB8-RNÁ helicase4 & 3.98 & & & * & a & a & a & a & a & a & a & a & b \\
\hline L205 & A0A1X7YHC0-30S ribosomal protein S2, chloroplastic a & n.d. & * & & & $a b$ & $\mathrm{ab}$ & a & $\mathrm{ab}$ & $a b$ & a & b & ab & $\mathbf{a}$ \\
\hline L127 & COPEC4-30S ribosomal protein S5 chloroplastic & 4.70 & * & & & $a b$ & $a b$ & $\mathrm{~b}$ & ab & a & b & $a b$ & $\mathrm{ab}$ & $a b$ \\
\hline L211 & P08530 - 30S ribosomal protein S8, chloroplastic & 3.27 & * & & & $a b$ & a & bc & $\mathrm{ab}$ & $a b$ & $\mathrm{bc}$ & $a b$ & a & c \\
\hline L137 & B4FR40-30S ribosomal protein S9 chloroplastic & n.d. & * & & & $a b$ & a & a & ab & b & a & $a b$ & $\mathrm{ab}$ & a \\
\hline L145 & B6UGL6—40S ribosomal protein S5 & 2.27 & * & * & & a & a & b & $\mathbf{a}$ & a & b & a & a & $\mathbf{b}$ \\
\hline L118 & A0A1D6P3R8-40S ribosomal protein S14 & 2.15 & & & * & $a b$ & a & $\mathrm{ab}$ & $\mathrm{ab}$ & $\mathrm{b}$ & $a b$ & a & $a b$ & $\mathrm{ab}$ \\
\hline L88 & B6SNQ7—40S ribosomal protein S16 & n.d. & & & * & $\mathrm{a}$ & a & $\mathrm{ab}$ & a & a & b & $a b$ & $\mathrm{a}$ & $\mathrm{ab}$ \\
\hline L171 & B6UF84-50S ribosomal protein L3 & 2.23 & * & & & $a b$ & a & $a b$ & $\mathrm{ab}$ & $a b$ & $a b$ & $a b$ & $\mathrm{ab}$ & $\mathrm{b}$ \\
\hline L195 & B6SK79-60S ribosomal protein L4 & 3.28 & * & & & $a b$ & a & $\mathrm{ab}$ & $a b$ & $\mathrm{ab}$ & $a b$ & $a b$ & $\mathrm{~b}$ & $\mathrm{~b}$ \\
\hline L216 & B6SHW0—60S ribosomal protein L6 & 2.33 & * & & & $\mathrm{a}$ & a & $\mathrm{ab}$ & $\mathrm{ab}$ & $\mathrm{a}$ & $a b$ & a & ab & b \\
\hline L126 & A0A1D6GM13-60S ribosomal protein L10 a & 2.40 & * & & & $a b$ & a & $\mathrm{ab}$ & $\mathrm{ab}$ & $a b$ & $a b$ & $a b$ & a & b \\
\hline L239 & B6TM00-60 ribosomal protein L14 & 2.66 & * & * & & $a b$ & a & bc & bc & bc & bc & $a b$ & ab & c \\
\hline L240 & B6SJH2—60S ribosomal protein L17 & 2.65 & * & & & a & a & $\mathbf{b}$ & a & a & b & a & a & b \\
\hline L15 & A0A1X7YIM9-RuBisCO large subunit-binding protein subunit alpha, chloroplastic a & 2.10 & * & & & c & a & bc & c & $\mathrm{abc}$ & c & c & ab & c \\
\hline L19 & B7ZZ42-Heat shock $70 \mathrm{kDa}$ protein 3 & 2.73 & & * & & $\mathrm{ab}$ & a & $a b$ & $\mathrm{ab}$ & $\mathrm{b}$ & $a b$ & $\mathrm{~b}$ & $\mathrm{ab}$ & $\mathrm{b}$ \\
\hline L64 & C3UZ63-HSP protein & 6.72 & * & & & abc & a & $\mathbf{c b}$ & $a b c$ & $\mathrm{abc}$ & bc & $a b$ & $a b c$ & c \\
\hline L70 & A0A1D6LIK1-Peptidyl-prolyl cis-trans isomerase & 2.04 & * & & & d & abcd & a & cd & abc & $a b$ & bcd & abcd & a \\
\hline L204 & B4FZZ2-Peptidyl-prolyl cis-trans isomerase & n.d. & * & & & b & $\mathbf{a}$ & a & c & a & a & bc & a & a \\
\hline L243 & A0A1D6FAW5-Peptidyl-prolyl cis-trans isomerase & n.d. & * & & & b & a & a & b & a & a & b & a & $\mathbf{a}$ \\
\hline L154 & B6TCN7-Threonine endopeptidase & 3.58 & & & * & $\mathrm{a}$ & $a b$ & $a b c$ & a & a & c & $a b$ & a & bc \\
\hline \multicolumn{15}{|c|}{ Stress response and other functions } \\
\hline L76 & A1XCI5-Lipoxygenase & 4.44 & * & * & & a & $\mathrm{a}$ & $\mathrm{a}$ & a & a & b & $\mathrm{a}$ & a & $\mathrm{ab}$ \\
\hline L189 & B6T2W9-Thylakoid lumenal $19 \mathrm{kDa}$ protein & 2.84 & & & * & b & a & $a b$ & a & $a b$ & $\mathrm{ab}$ & $\mathrm{ab}$ & a & $a b$ \\
\hline L201 & B6SU36-Thylakoid lumenal $16.5 \mathrm{kDa}$ protein & 10.3 & & & * & a & ab & b & $\mathrm{ab}$ & $\mathrm{a}$ & $a b$ & $\mathrm{ab}$ & $\mathrm{ab}$ & $\mathrm{ab}$ \\
\hline L143 & B6T7W8-Cortical cell-delineating protein & 2.57 & * & & & $a b$ & a & b & $a b$ & $a b$ & $\mathrm{~b}$ & $a b$ & a & b \\
\hline L180 & K7UGI3-Putative thioredoxin superfamily protein & 4.52 & * & & & abc & a & bcd & abcd & ab & $\mathrm{cd}$ & $a b$ & a & $\mathrm{d}$ \\
\hline L208 & C4J9Y2-Aluminum-induced protein homolog1 & 10.5 & * & & & $a b$ & a & $\mathbf{b}$ & $\mathrm{a}$ & $\mathrm{a}$ & $\mathrm{ab}$ & $a b$ & $\mathrm{a}$ & $a b$ \\
\hline
\end{tabular}




\subsection{Proteomic Changes Involved in Nitrogen (N) Assimilation and Amino Acid Metabolism}

The proteomic analysis showed that the nutritional treatments induced changes in the levels of Ferredoxin-Nitrite Reductase (Fd-NiR) in roots (R74, Figure 4) and in leaves (L149, Figure 5), with trends similar to the contents of $\mathrm{NO}_{3}{ }^{-}$in the organ (Figure 2). This was particularly evident in (n) plants, in which Fd-NiR reached the maximum level after $30 \mathrm{~h}$ and $54 \mathrm{~h}$ in roots and leaves, in conjunction with the peak of $\mathrm{NO}_{3}{ }^{-}$accumulation. Moreover, the Fd-NiR levels in (na) plants (Figures 4 and 5) suggest that $\mathrm{NO}_{3}{ }^{-}$reduction was sustained even in the presence of $\mathrm{NH}_{4}{ }^{+}$.

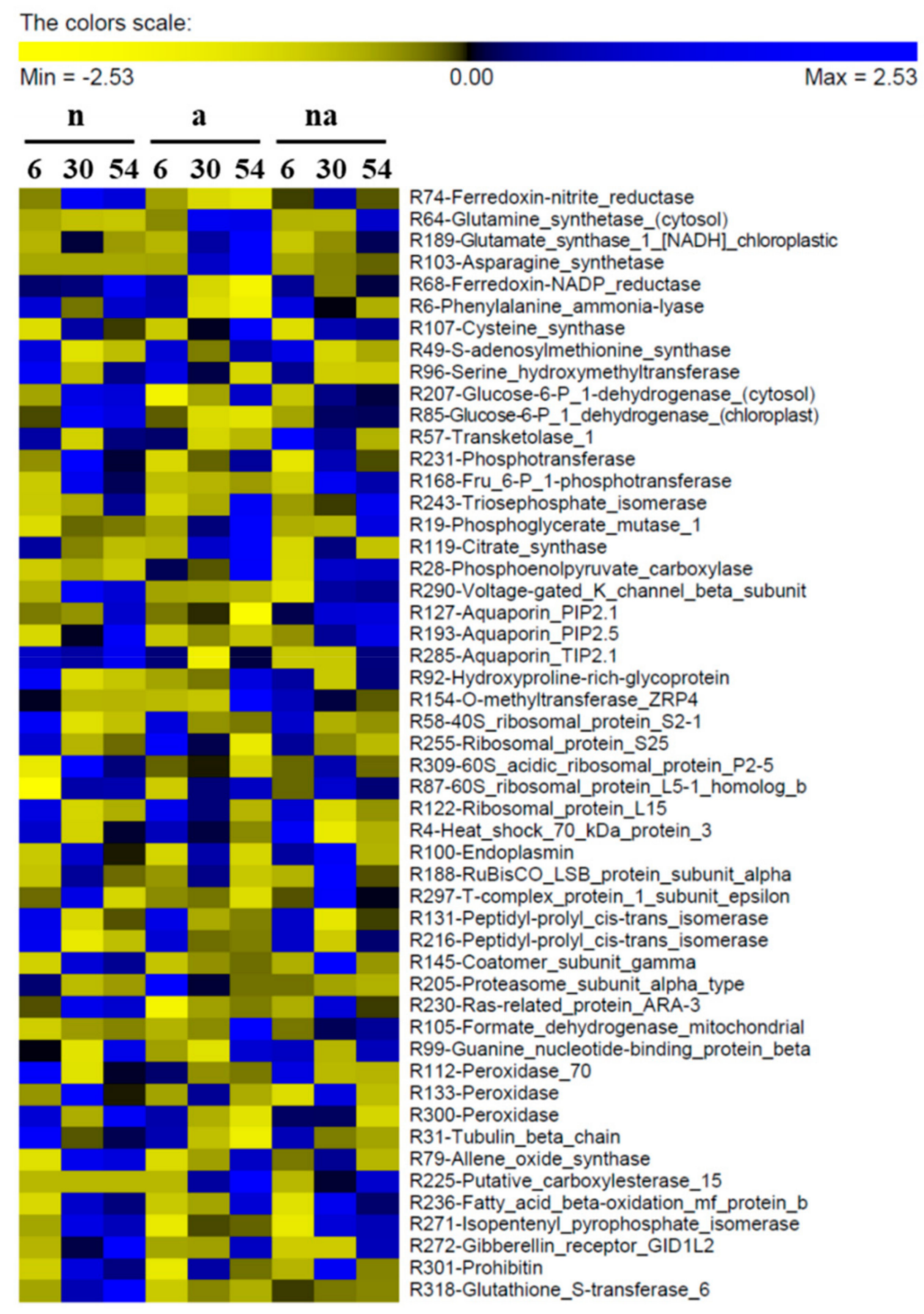

Figure 4. Abundance of the differentially accumulated proteins in maize roots. Maize plants were exposed for 6, 30, and $54 \mathrm{~h}$ to the presence of $5 \mathrm{mM} \mathrm{NO}_{3}^{-}$(n), $5 \mathrm{mM} \mathrm{NH}_{4}{ }^{+}$(a), and $2.5 \mathrm{mM} \mathrm{NO}_{3}^{-}+$ $2.5 \mathrm{mM} \mathrm{NH}_{4}{ }^{+}$(na). The image was obtained by means of the PermutMatrix graphical interface after Z-score normalization of the averages of protein Spectrum Intensity \% (\%SI, $n=3)$. Each colored cell represents the average of the \%SI according to the color scale.

Considering that plastid Fd-NADP ${ }^{+}$reductase (FNR, R68, Table 3) reduces the Fd-like electron carrier for $\mathrm{NiR}$ [31], it is of interest that FNR and Fd-NiR showed similar profiles in roots (Figure 4). These results confirmed that both enzymes are strictly coordinated and take part in the "root primary response to $\mathrm{NO}_{3}{ }^{-\prime \prime}[29,32]$. 
On the contrary, the trends observed for two glutamine synthetases (GS) were dissimilar in roots and leaves, probably because the two enzymes were different isoforms with well-known specific roles. In leaves, the enzyme (L177, Table 4) belongs to the chloroplast GS2 type (92.8\% of identity with GS2 P25462) which plays a pivotal role in $\mathrm{NO}_{3}{ }^{-}$assimilation. Its decrease in the course of time (Figure 5) could be due to leaf age and/or to amino acid accumulation, as previously observed [33]. Instead, in roots GS was identified as the cytosolic GS1-1 isoform (R64, Table 3, 99.7\% of identity with GS1-1 P38559) that surged up in plants exposed to $\mathrm{NH}_{4}{ }^{+}$(a, na, Figure 4), confirming its role in $\mathrm{NH}_{4}^{+}$ assimilation [27].

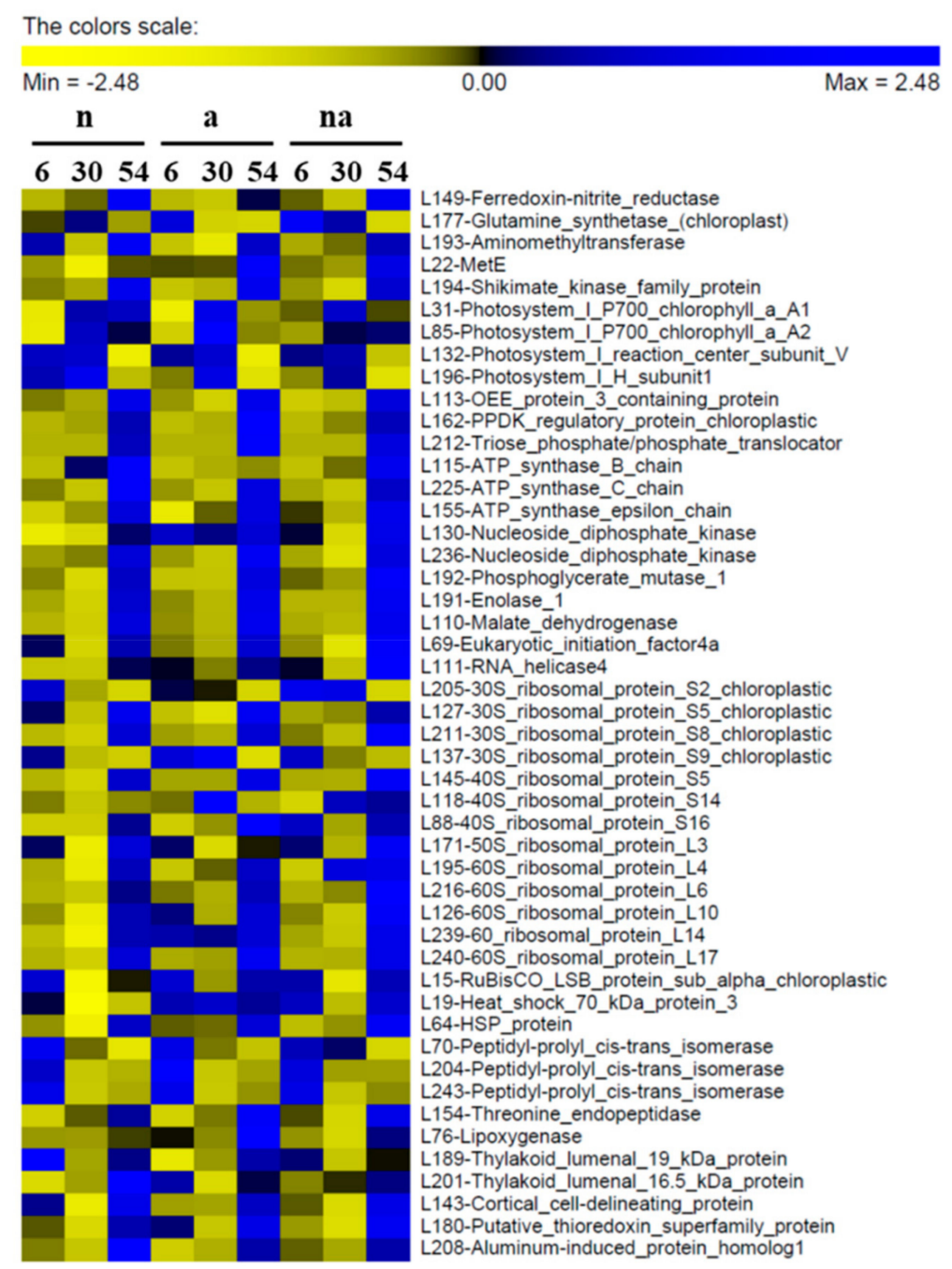

Figure 5. Abundance of the differentially accumulated proteins in maize leaves. Maize plants were exposed for 6, 30, and $54 \mathrm{~h}$ to the presence of $5 \mathrm{mM} \mathrm{NO}_{3}^{-}$(n), $5 \mathrm{mM} \mathrm{NH}_{4}{ }^{+}$(a), and $2.5 \mathrm{mM} \mathrm{NO}_{3}{ }^{-}+$ $2.5 \mathrm{mM} \mathrm{NH}_{4}{ }^{+}$(na). The image was obtained by means of the PermutMatrix graphical interface after Z-score normalization of the averages of protein Spectrum Intensity \% (\%SI, $n=3)$. Each colored cell represents the average of the \%SI according to the color scale.

The concurrent increases of GS1, of the glutamate synthase 1 [NADH] chloroplastic (R189, Table 3) and of the amino acid levels in roots of (a) plants (Figures $2 \mathrm{~F}$ and 4 ) indicated a relevant induction of $\mathrm{NH}_{4}{ }^{+}$assimilation. Interestingly, all these traits were lower in the roots of the (na) plants, even if the $\mathrm{NH}_{4}{ }^{+}$content was higher (Figures $2 \mathrm{C}$ and 4), confirming that the copresence of $\mathrm{NO}_{3}{ }^{-}$could somehow reduce the need to quickly assimilate the $\mathrm{NH}_{4}{ }^{+}$ions (see below). 
This hypothesis was further supported by the profile of the asparagine synthetase (AS), the enzyme that catalyzes the ATP-dependent synthesis of asparagine by the transfer of the amino group from glutamine to aspartate. In several plant species, some members of the AS gene family are upregulated by increases in the levels of amino acids and/or of internal $\mathrm{NH}_{4}{ }^{+}$, suggesting that AS could contribute to its assimilation during nutritional stress conditions [34]. In our study, one AS (R103) was greatly induced in the roots of the $\mathrm{NH}_{4}{ }^{+}$-supplied plants (a), while it was almost undetectable in plants exposed to nitrate or to co-provision (n, na, Figure 4), confirming that the presence of $\mathrm{NO}_{3}{ }^{-}$ could reduce the stress induced by $\mathrm{NH}_{4}{ }^{+}$accumulation in roots.
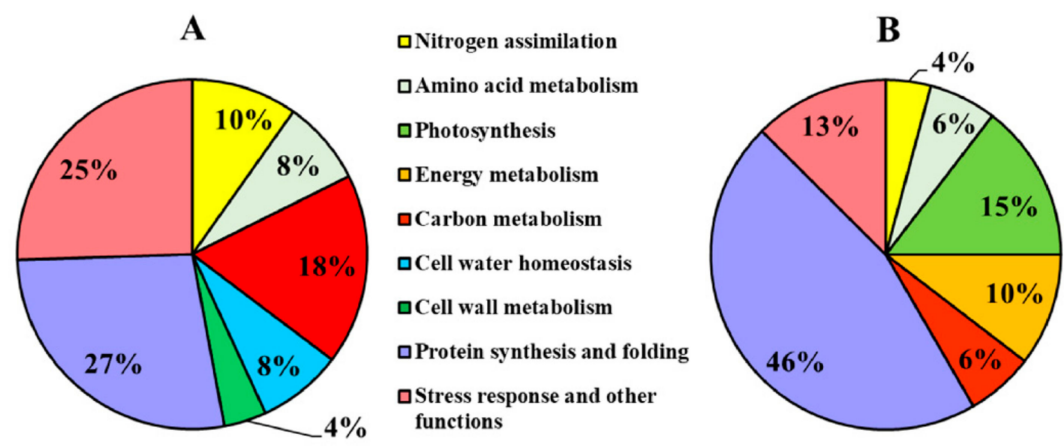

Figure 6. Functional distribution of the differentially accumulated proteins in maize plants. The proteins differentially accumulated were grouped in classes according to literature and GeneBank. (A) Proteins differentially accumulated in roots; (B) proteins differentially accumulated in leaves. The functional distribution indicates the percentage of each class as compared to the total number of proteins differentially accumulated.
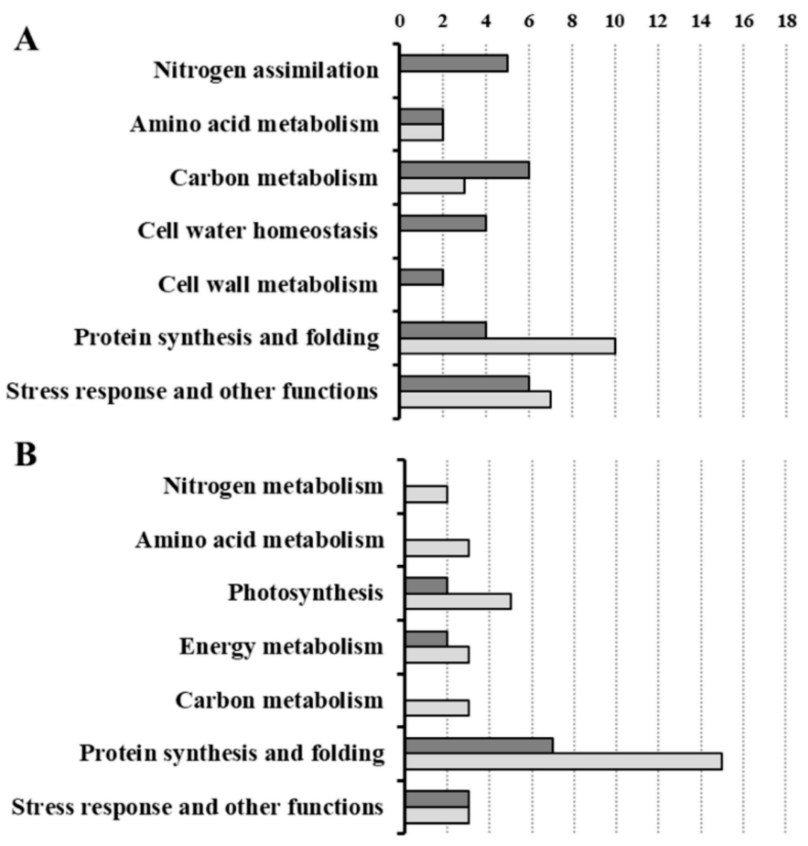

$\square$ Specifically influenced by the $N$ source $\square$ Not influenced by the $N$ source

Figure 7. Classification of the differentially accumulated proteins according to the main source of variation in roots (A) and in leaves (B). The proteins differentially accumulated, sorted in functional classes, are categorized in two groups: proteins whose changes were specifically related to the $\mathrm{N}$ source (dark grey bars) and proteins whose changes were not related to $\mathrm{N}$ sources, but to other factors, such as time and the total $\mathrm{N}$ availability (light grey bars). 
In this context, it is important to note that it was proposed that glutamate dehydrogenase (GDH) could contribute to the assimilative process when plants are exposed to an excessive $\mathrm{NH}_{4}{ }^{+}$nutrition [2]. Our proteomic analysis revealed that in roots GDH did not change in abundance during any treatment (R104, Supplementary Table S1). This observation is in agreement with the fact that the $\mathrm{NH}_{4}{ }^{+}$content in roots never reached levels associated with toxicity in maize [22,27], but it does not exclude the idea that the enzyme could have relevant roles at higher $\mathrm{NH}_{4}{ }^{+}$inputs or during longer exposures.

On the whole, the proteomic analysis provided evidence that co-provision could also have positive effects on plants' growth because of the ability of $\mathrm{NO}_{3}{ }^{-}$to change the balance between the quota of $\mathrm{NH}_{4}{ }^{+}$drained by assimilation and the quota of $\mathrm{NH}_{4}{ }^{+}$delivered to vacuole storage.

At the same time, the proteomic analysis revealed changes in the levels of enzymes involved in amino acid metabolism in roots and leaves, most of which were not specifically related to the $\mathrm{N}$ source (Figure 7). In leaves, enzymes involved in glycine (L193), methionine (L22), and aromatic amino acid (L194) metabolisms increased in abundance over time (Table 4, Figure 5), in agreement with a raising of the leaf anabolic processes after the $\mathrm{N}$ inputs. In roots, the members of this class were differently affected as regards both trends and sources of variation. The levels of phenylalanine ammonia-lyase (PAL) decreased in plants exposed to $\mathrm{NH}_{4}{ }^{+}$, both during (a) and (na) treatment (R6, Table 3, Figure 4). These data suggest that, similarly to $\mathrm{NO}_{3}{ }^{-}[20,35]$, the $\mathrm{NH}_{4}{ }^{+}$contents could also exert negative feedback at the enzyme level, probably to reduce additional release of the cation via PAL activity.

\subsection{Changes in Proteins Involved in Photosynthesis, Energy, and Carbon Metabolism}

The leaf proteome was characterized by DPs involved in photosynthesis and energy metabolism, many of which were mainly influenced by time and/or by the total $\mathrm{N}$ availability (Figures 6 and 7). The increases in the Photosystem I P700 chlorophyll a apoproteins A1 and A2 (L31, L85, Table 4, Figure 5), which are the large subunits PsaA and PsaB that form the core of the Photosystem I complex (PSI) [36], support the hypothesis that a general increase of PSI functionality occurred to sustain $\mathrm{N}$ assimilation.

On the other hand, in leaves in all conditions ( $\mathrm{n}, \mathrm{a}$, and na) at $54 \mathrm{~h}$, several events occurred at once, along with the increase in $\mathrm{NH}_{4}{ }^{+}$and amino acid content (Figure 2). Firstly, it was possible to observe a decrease in abundance of the PSI reaction center subunit V (L132, also known as PsaG) and of the PSI H subunit1 (L196), which are involved in the interaction of the PSI with the light harvesting complexes I (LCHI) and LCHII, respectively [36]. In addition, it was possible to observe an increase of the oxygen evolving enhancer (OEE) protein 3 (L113), which belongs to the photosystem subunit Q (PsbQ) family involved in stabilizing the PSII-LCHII complex [37]. These results support the hypothesis that, at $54 \mathrm{~h}$, the leaves went through a modulation of the energy balance between the two Photosystems (i.e., a transition from State II to State I) that promoted PSII functionality and linear electron flow. This condition corresponds to an optimization of the photosynthetic machinery to generate both adenosine triphosphate (ATP) and reduced form of nicotinamide adenine dinucleotide phosphate (NADPH) required by the Calvin Cycle [38]. It coincided with an increase of three subunits of chloroplast ATP synthase (L115, L225, and L155) and of the pyruvate phosphate dikinase regulatory protein (L162), which regulates $\mathrm{CO}_{2}$ fixation in $\mathrm{C}_{4}$ plants [39]. Finally, the concomitant increases of the triose phosphate/phosphate translocator (L212), which mediates the export of fixed carbon from chloroplast to cytosol [40], of two nucleoside diphosphate kinases (L130 and L236), cytosolic enzymes involved in balancing between the pools of ATP and the nucleosides [41], as well as of two glycolytic enzymes (L192 and L191) and of the mitochondrial malate dehydrogenase (L110) suggested a general upsurge in energy production and in respiratory metabolism (Table 4, Figure 5). On the whole, considering that in plants the maintenance of low levels of $\mathrm{NH}_{4}{ }^{+}$in tissues is one of the main strategies to avoid metabolic stresses [11], it is possible to propose that, at $54 \mathrm{~h}$, the $\mathrm{NH}_{4}{ }^{+}$accumulation in leaves led to an increment of the photosynthetic machinery and of $\mathrm{C}$ metabolism to sustain the synthesis of amino acids. In this regard, the high content of reducing sugars in the roots of the (a) plants at $54 \mathrm{~h}$ could indicate that a massive allocation of photoassimilates in this organ occurred when $\mathrm{NH}_{4}{ }^{+}$was 
provided as the sole N nutrient (Figure 3C). This response, curiously absent in co-provision, could be one of the causes contributing to the slower leaf growth (Figure 1B).

In roots, $\mathrm{C}$ metabolism was instead more specifically affected by the kind of $\mathrm{N}$ source (Table 3 , Figure 7). Seeing that the oxidative pentose pathway is induced by $\mathrm{NO}_{3}{ }^{-}$, as indicated in maize root plastids [42] and by transcriptomics in Arabidopsis roots [30], it is important to analyze the changes of two isoforms of glucose-6-phosphate 1-dehydrogenase (G6PDH, Table 3). The first one, the R207, which is probably a cytosolic form ( $80 \%$ of identity with P37830, a cytoplasmic isoform of S. tuberosum), increased with time. However, the second one, the R85, which belongs to the plastid protein cluster, showed a higher level in (n) and (na) plants, and it almost disappeared in (a) roots after $54 \mathrm{~h}$ (Table 3, Figure 4). These results are in agreement with the fact that in barley (H. vulgare L.) the cytosolic G6PDH activity seems to be correlated with general growth processes, while the plastidic one is induced to a higher extent by $\mathrm{NO}_{3}{ }^{-}$than by $\mathrm{NH}_{4}{ }^{+}$[43].

Similarly, some root glycolytic enzymes were differently affected by the nutritional treatments (Table 3). The pyrophosphate-fructose 6-phosphate 1-phosphotransferase (R168, also known as phosphofructokinase) was induced by $\mathrm{NO}_{3}{ }^{-}$in (n) and (na) roots (Figure 4). Although some studies exclude a key role for this enzyme in the control of glycolysis [44], in our opinion, this response deserves further investigation. Moreover, since changes in the phosphoglycerate mutase levels seem to have dramatic effects on metabolism [44], the increases in abundance of this enzyme (R19), together with citrate synthase (R119) and PEP carboxylase (R28) suggested an upsurge of respiratory metabolism in roots of the (a) plants at $54 \mathrm{~h}$ (Figure 4). With this in view, these results confirm that in roots of $\mathrm{NH}_{4}{ }^{+}$-fed plants the PEP carboxylase could play an important anaplerotic role for TCA replenishment, as previously proposed by several authors $[2,10,45]$. Taken together, these results provide new evidence that $\mathrm{NO}_{3}{ }^{-}$and $\mathrm{NH}_{4}{ }^{+}$have different effects on $\mathrm{C}$ metabolism in roots, according to different requirements for reducing power and $\mathrm{C}$ skeletons.

Overall, this proteomic study highlights the different metabolic roles for roots and leaves and it allows us to propose novel molecular determinants involved in the adaptation of plants to different $\mathrm{N}$ sources.

\subsection{Root Proteomic Changes Involved in Cell Water Homeostasis and Cell Wall Metabolism}

The root proteomic profile was characterized by DPs involved in cell water homeostasis and cell wall metabolism that were all specifically affected by the $\mathrm{N}$ source (Table 3, Figures 6A and 7A). Considering the interactions between $\mathrm{K}$ and $\mathrm{N}$ nutrition on ion uptake, transport, and assimilation in plants [46], it is appropriate to highlight that the $\beta$ subunit of a V-gated $\mathrm{K}^{+}$channel (R290, Table 3) was specifically induced in (n) plants, where it reached its highest abundance after $30 \mathrm{~h}$. Although to lesser extent, this effect was also appreciable in roots of the (na) plants but it was absent in the $\mathrm{NH}_{4}{ }^{+}$-fed plants (Figure 4). This observation could be associated with the fact that, at high external concentrations, the acquisition rates of $\mathrm{NO}_{3}{ }^{-}$and $\mathrm{K}^{+}$are often positively correlated ([46] and references therein), a relation that in barley roots was recently attributed to the stimulative effect of $\mathrm{NO}_{3}{ }^{-}$on the $\mathrm{K}^{+}$low-affinity influx system [47]. Whether this effect derives from an improvement in cell electrical balance and/or from a regulative molecular mechanism is as yet an unresolved question, which deserves future physiological and molecular studies.

The $\mathrm{N}$ sources also differently affected the accumulation of some aquaporins located at both plasma membrane (PIP) and tonoplast (TIP) (Table 3). In particular, PIP2.1 (R127) maintained the highest levels in (n) and (na) roots but decreased by half at $54 \mathrm{~h}$ in (a) plants, while PIP2-5 (R193) was specifically accumulated in roots exposed to $\mathrm{NO}_{3}{ }^{-}$(n and na, Figure 4). Several authors have proposed that the increment in root hydraulic conductivity during $\mathrm{NO}_{3}{ }^{-}$exposure involves changes in PIP functionality [48]). However, a study conducted in maize indicated that $\mathrm{NO}_{3}{ }^{-}$induces an increase in root hydraulic conductivity, but it does not correlate with any changes in the expression of the aquaporin genes, at least within $4 \mathrm{~h}$ of treatment [49]. It seems likely that the discrepancy between that study and our proteomic profiles derives from different exposure timing. For instance, it is 
possible that $54 \mathrm{~h} \mathrm{NO}_{3}{ }^{-}$accumulation in root tissues (Figure 2A) could have had effects on aquaporin abundances. Moreover, TIP2.1 (R285) is very similar to the aquaporin AtTIP2-3 (77\% identity with Q9FGL2) which in Arabidopsis is involved in $\mathrm{NH}_{3}$ transport into the vacuole [50]. This protein was more abundant in the roots of (n) plants (Figure 4), suggesting that high $\mathrm{NH}_{4}{ }^{+}$external inputs could exert inhibitory effects on the channel.

Although future studies are needed for a conclusive verification, these observations allow us to propose that the presence of $\mathrm{NO}_{3}{ }^{-}$might promote a higher accumulation of $\mathrm{K}^{+}$channels and aquaporins in roots, maybe acting as an osmolyte. Considering that both protein families are involved in the $\mathrm{NH}_{4}{ }^{+} \mathrm{NH}_{3}$ transport [11], it is possible to conceive some relationship between their induction by $\mathrm{NO}_{3}{ }^{-}$and the highest $\mathrm{NH}_{4}{ }^{+}$content in roots in co-provision (Figure 2D).

Furthermore, several authors have proposed links among aquaporin expression, cell water potential, and cell expansion in growing tissues [48]. Starting from these considerations, it is interesting to correlate the changes in aquaporins with the changes of the DPs involved in cell wall metabolism in roots. The proteomic analysis revealed that a hydroxyproline-rich glycoprotein dramatically decreased in abundance after $54 \mathrm{~h}$ of (n) nutrition (R92), while O-methyltransferase ZRP4 (R154) was specifically accumulated after $54 \mathrm{~h}$ of (a) exposure (Table 3, Figure 4). Since in maize roots these proteins are associated with the lignification and suberization of the secondary cell wall [51,52], these results could suggest that $\mathrm{NO}_{3}{ }^{-}$, in contrast with $\mathrm{NH}_{4}{ }^{+}$, induced changes sustaining root growth and development. This hypothesis is also consistent with the differences in root growth in (n) and (a) plants (Figure 1A). This topic deserves future investigation, which could also contribute to elucidating the molecular mechanisms underlying the differences in root morphology between $\mathrm{NO}_{3}{ }^{-}$- and $\mathrm{NH}_{4}{ }^{+}$-fed plants.

\subsection{Proteomic Changes Related to Protein Synthesis and Folding}

The "protein synthesis and folding" was the major functional class both in roots and leaves, accounting for $27 \%$ and $46 \%$ of the DPs, respectively (Figure 6). In both cases, most of them were not specifically affected by the $\mathrm{N}$ source (Figure 7), probably because the modulation of protein synthesis was mainly associated with plant growth after exposure to high $\mathrm{N}$ availabilities. This class encompasses many kinds of proteins, among which are an initiation factor (L69), three heat shock proteins (R4, L19, and L64), and an endopeptidase (L154) (Tables 3 and 4).

Ribosomal proteins accounted for $36 \%$ and $59 \%$ of the category in roots and leaves, respectively, and, in particular, in leaves they were identified as both chloroplastic $(30 \%)$ and cytosolic $(70 \%)$ members (Tables 3 and 4). In general, the trends observed were very specific for each ribosomal DPs (Figures 4 and 5), probably due to a modulation of the ribosome composition. This conclusion is in agreement with a previous study showing that the replenishment of $\mathrm{NO}_{3}{ }^{-}$in $\mathrm{N}$-starved Arabidopsis seedlings induces changes in the expression of more than 100 genes encoding ribosomal proteins [53]. However, it is very difficult to draw conclusions about the biochemical meanings of these changes because of the complexity that characterizes the plant ribosome. Plant ribosomes are composed of a large number of heterogeneous proteins grouped into many families, currently numbering 80 in the Arabidopsis genome [54], encoded by several gene paralogs, with specific developmental roles, for which transcriptional regulation is still unclear $[55,56]$. To unravel these aspects is far beyond the aims of this work, but we believe that the information provided by this proteomic profiling can be useful for future studies dealing with this fundamental issue of plant biology.

Several peptidyl-prolyl cis-trans isomerases (PPIases) of the cyclophilin-type (Cyp), namely, two in the roots (R131 and R216, Table 3) and three in leaves (L70, L204, and L243, Table 4) decreased in abundance in all the nutritional treatments over time (Figures 4 and 5). The PPIases catalyse the cis-trans isomerization of prolyl bonds in polypeptide chains avoiding the accumulation of misfolded proteins, both during de novo synthesis and in restructuring of mature polypeptides [57]. In plants, one of the first Cyp was discovered in maize and its function was related to responses to abiotic stresses [58]. Moreover, in humans, Cyp18 seems to be involved in the elimination of damaged proteins accumulated under oxidative stress [59]. Since oxidative stress and protein synthesis alterations are 
often associated with nutrient deficiency, it is possible that the decline of these proteins was due to the exposure of the plants to high $\mathrm{N}$ inputs. This result supports the hypothesis that the Cyp proteins could contribute to plant defense responses during nutritional shortages.

Summing up, this proteomic profiling provides new evidence that the modulation of protein synthesis is a crucial and multi-faceted element in plant adaptation to $\mathrm{N}$ availability, which requires coordination of several protein families.

\subsection{Stress Responses and Other Functions}

The DPs related to stress responses and other functions allow us to point out two interesting differences between (a) and (na) plants, which are therefore probably related to the presence of $\mathrm{NO}_{3}{ }^{-}$ in co-provision. In particular, after $54 \mathrm{~h}$ of treatment, (a) plants were characterized by a relevant upsurge in abundance of formate dehydrogenase (R105) in roots and of lipoxygenase (L76) in leaves (Tables 3 and 4, Figures 4 and 5). Both increases were not significant in (na) plants, indicating that these responses were reduced by $\mathrm{NO}_{3}{ }^{-}$availability although the $\mathrm{NH}_{4}{ }^{+}$content was higher in roots and similar in leaves (Figure 2).

Formate dehydrogenase is a mitochondrial enzyme that catalyzes the $\mathrm{NAD}^{+}$dependent oxidation of formate to $\mathrm{CO}_{2}$ [60]. In non-photosynthetic tissues, one of the most plausible routes for formate production is the catabolism of serine and glycine [61]. It is conceivable that this metabolic condition may have occurred in response to the high accumulation of amino acids in roots during $\mathrm{NH}_{4}{ }^{+}$nutrition, a trait that was less pronounced in co-provision (Figure 2). Moreover, in plants the induction of formate dehydrogenase is often induced by several abiotic stress, such as darkness and anoxia. Recently it was related with aluminium toxicity, caused by a metal cation whose detoxification shows several similarities with plant responses to $\mathrm{NH}_{4}{ }^{+}$, such as the production of organic acids and the vacuolar sequestration of this cation [62]. Considering that one-carbon metabolism could have roles in sustaining amino acid biosynthesis in non-photosynthetic tissues, our results suggest that formate dehydrogenase could be participating in mechanisms of tolerance to $\mathrm{NH}_{4}{ }^{+}$in maize plants.

Plant lipoxygenases (LOX) are involved in polyunsaturated fatty acid and membrane metabolisms, but it has also been proposed that these enzymes could be accumulated as vegetative storage proteins in leaves, as observed in soybean (Glycine max L.) in response to sink limitation [63]. In one of our previous proteomic studies in maize, we were able to establish that the LOX identified in this analysis (codified by ZmLOX10) was accumulated in the leaf in response to high $\mathrm{NO}_{3}{ }^{-}$supply (i.e., $10 \mathrm{mM} \mathrm{NO}_{3}{ }^{-}, 30 \mathrm{~h}$ ) [20]. Although it is not possible to exclude an involvement in the protection of stress induced by $\mathrm{NH}_{4}{ }^{+}$, all together these results support the hypothesis that $\mathrm{LOX}$ accumulation in the leaf could be a way to store N, a new and intriguing role of LOX in cereal crops.

In addition, three root peroxidases of the class III (Prxs) showed significant changes in abundance (R112, R133, and R300, Table 3, Figure 4). Since Prxs play several roles in plants [64], it is difficult to assign specific biochemical meaning to these results. However, the relations among Prxs, cell wall metabolism in roots and $\mathrm{N}$ nutritional status in plants seem to be worthy of further investigation. Similarly, it is interesting to note that the broad class of "other functions" includes several proteins localized in plant cell organelles, such as a peroxisomal fatty acid beta-oxidation multifunctional protein (R236), a mitochondrial prohibitin (R301), and two thylakoid luminal $19 \mathrm{kDa}$ and $16.5 \mathrm{KDa}$ proteins (L189 and L201) with unknown function (Tables 3 and 4). This observation leads us to assume that, within the next few years, subcellular proteomics studies will be very useful to obtain novel information about the roles played by cell organelles in plant adaptation to total $\mathrm{N}$ availability as well as to different $\mathrm{N}$ sources. 


\section{Materials and Methods}

\subsection{Plant Material and Nutritional Treatments}

Maize (Zea mays L.) seeds of the line PR33A46 (Pioneer Hi-Bred Italia ${ }^{\circledR}$, Gadesco Pieve Delmona, $\mathrm{CR}$, Italy) were germinated in the dark at $26^{\circ} \mathrm{C}$ for $72 \mathrm{~h}$. Seedlings were then grown by a hydroponic system in a growth chamber with a $16 / 8 \mathrm{~h}$ day/night regime, at $26 / 22{ }^{\circ} \mathrm{C}$, constant relative humidity of $65 \%$, and PPFD of $500 \mu \mathrm{mol} \cdot \mathrm{m}^{-2} \cdot \mathrm{s}^{-1}$. After $48 \mathrm{~h}$ of incubation in $4 \mathrm{mM} \mathrm{CaSO}_{4}$, the plants were transferred into a growth solution with low $\mathrm{N}$ input $\left(1 \mathrm{mM} \mathrm{KNO}, 2 \mathrm{mM} \mathrm{K}_{2} \mathrm{SO}_{4}, 0.875 \mathrm{mM} \mathrm{KH}_{2} \mathrm{PO}_{4}\right.$, $0.5 \mathrm{mM} \mathrm{MgSO}_{4}, 0.4 \mathrm{mM} \mathrm{CaSO}_{4}, 62.5 \mu \mathrm{M}\left(\mathrm{NH}_{4}\right)_{2} \mathrm{SO}_{4}, 60 \mu \mathrm{M}$ Fe-EDTA, $25 \mu \mathrm{M} \mathrm{KCl}, 12.5 \mu \mathrm{M} \mathrm{H}_{3} \mathrm{BO}_{3}$, $1 \mu \mathrm{M} \mathrm{MnSO}_{4}, 0.25 \mu \mathrm{M} \mathrm{CuSO}_{4}, 0.25 \mu \mathrm{M} \mathrm{ZnSO}_{4}, 0.25 \mu \mathrm{M} \mathrm{Na}_{2} \mathrm{MoO}_{4}, \mathrm{pH}=6.1$ ). After six days, at the beginning of the day ( $\mathrm{t} 0$ ), the plants were transferred into new growth solutions in which $\mathrm{N}$ availability was changed according to the following treatments (abbreviated by letters in brackets): (i) $5 \mathrm{mM} \mathrm{NO}_{3}{ }^{-}$(n); (ii) $5 \mathrm{mM} \mathrm{NH}_{4}^{+}$(a); (iii) $2.5 \mathrm{mM} \mathrm{NO}_{3}{ }^{-}+2.5 \mathrm{mM} \mathrm{NH}_{4}{ }^{+}$(na). All the solutions were balanced with $\mathrm{K}_{2} \mathrm{SO}_{4}$ and continuously aerated by electric pumps. The plants were sampled at $\mathrm{t} 0$ and after 6,30 , and $54 \mathrm{~h}$ of treatment. Roots were rinsed with water and blotted with paper towels. Roots and leaves were separately collected, weighed and immediately frozen in liquid $\mathrm{N}_{2}$. Each biological sample was composed of roots or leaves collected from four plants. Samples were stored at $-80^{\circ} \mathrm{C}$. The significance of the changes in the biomass accumulation in roots and in leaves $(n=8)$ was assessed by the ANOVA test $(p<0.05$, Tukey post hoc method).

\subsection{Determination of the Contents of Nitrate, Ammonium, Amino Acids, Sucrose and Reducing Sugars}

Nitrate was extracted from leaf and root samples as previously described [20], and measured according to Cataldo et al. [65].

The contents of $\mathrm{NH}_{4}{ }^{+}$in roots and leaves were measured by the $o$-phthalaldehyde (OPA) method, as described by Coskun and coworkers [66]. Briefly, samples were powdered in liquid $\mathrm{N}_{2}$, homogenized in 5 volumes of ice-cold $10 \mathrm{mM}$ formic acid (FA), and centrifuged at 14,000 $\mathrm{g}$ for $10 \mathrm{~min}$ at $4{ }^{\circ} \mathrm{C}$. The supernatants were filtered by Millipore Millex HC cartridges $(0.45 \mu \mathrm{m})$. An aliquot of the extract was added to $3 \mathrm{~mL}$ of OPA reagent $\left(100 \mathrm{mM} \mathrm{KH}_{2} \mathrm{PO}_{4}, 100 \mathrm{mM} \mathrm{K} \mathrm{HPO}_{4}, 3.75 \mathrm{mM} \mathrm{OPA}\right.$, $2 \mathrm{mM}$ 2-mercaptoethanol (2-ME), $\mathrm{pH}=7$ ). After incubation for $30 \mathrm{~min}$ in the dark, the sample absorbance was determined at $410 \mathrm{~nm}$.

Amino acids, reducing sugars and sucrose were extracted from leaf and root samples as previously described [20]. Amino acid concentration was measured by the ninhydrin method [67], while the contents of sucrose and reducing sugars were determined according to Nelson [68]. The total content of $\mathrm{N}$ in roots and leaves was calculated as the sum of the inorganic $\mathrm{N}$ (derived from the contents of $\mathrm{NO}_{3}{ }^{-}$ and $\mathrm{NH}_{4}{ }^{+}$) plus the organic $\mathrm{N}$ (derived from the contents of amino acids and proteins, applying the conversion factor of 6.25 that is generally used in corn [69]). All of the analyses were replicated on three independent biological samples $(n=3)$ and compared by the ANOVA test $(p<0.05$, Tukey post hoc method).

\subsection{Protein Extraction, Gel Electrophoresis and in-Gel Digestion}

Frozen samples of roots or leaves were powdered in liquid $\mathrm{N}_{2}$ using a mortar and pestle and an aliquot of $200 \mathrm{mg}$ was suspended in 3 volumes of extraction buffer $(150 \mathrm{mM}$ Tris- $\mathrm{HCl} \mathrm{pH} \mathrm{6.8,}$ $10 \%(w / v)$ glycerol, $2 \%(w / v)$ sodium dodecyl sulfate (SDS), $2 \%(v / v)$ 2-ME, $0.1 \mathrm{mg} \cdot \mathrm{mL}^{-1}$ Pefablock $^{-}$ (Fluka)). Samples were vortexed for $10 \mathrm{~min}$ at room temperature and incubated for $30 \mathrm{~min}$ at $90{ }^{\circ} \mathrm{C}$. After centrifugation at $14,000 \times g$ for $15 \mathrm{~min}$, the collected supernatants were recentrifuged at $14,000 \times g$ for $5 \mathrm{~min}$. The supernatants were stored at $-80{ }^{\circ} \mathrm{C}$ until further use. Protein concentration was measured by 2-D Quant Kit (GE Healthcare, Milan, Italy).

Protein samples were colored with traces of bromophenol blue and aliquots of $15 \mu \mathrm{g}$ were purified by partial 1D SDS-PAGE (1-dimensional SDS-polyacrylamide gel electrophoresis) conducted on 16\% $(w / v)$ polyacrylamide gel [70], monitoring by the Full-Range Rainbow Markers (Mr 12 000-225 000) 
(GE Healthcare). Briefly, the run was conducted applying $60 \mathrm{mV}$ for $30 \mathrm{~min}$ until protein samples completely entered in the running gel. The gels were then incubated for $1 \mathrm{~h}$ in fixing solution $(10 \%(v / v)$ acetic acid, $50 \%(v / v)$ methanol), stained for $1 \mathrm{~h}$ with Coomassie Brilliant Blue (CBB) $(0.1 \%(w / v)$ CBB $\mathrm{R}-250,10 \%(v / v)$ acetic acid), and destained in 10\% (v/v) acetic acid.

The portion of gel containing proteins was excised and subjected to tryptic digestion. In-gel digestion was performed as previously described [20] with the following refinements. The volumes of solutions were adjusted to completely cover all gel samples (previously cut into 12 portions), and each sample was treated with $3 \mu \mathrm{g}$ of trypsin (V5111, Promega, Madison, WI, USA). The extracted peptides were suspended in $0.1 \%(v / v)$ formic acid (FA). All of the procedures were replicated on three independent biological samples $(n=3)$.

\subsection{Mass Spectrometry Analysis}

All mass spectrometry experiments were conducted on an Agilent 6520 Q-TOF mass spectrometer equipped with an HPLC Chip Cube source driven by a 1200 series nano/capillary LC system (Agilent Technologies, Cernusco Sul Naviglio, MI, Italy)). Both systems were controlled by a MassHunter Workstation (version B.02.01, B2116.20; Agilent Technologies). Chromatography was performed into Polaris-HR-Chip-3C18 (Agilent Technologies), consisted of a 360-nL trap column and a $75 \mu \mathrm{m} \times 150-\mathrm{mm}$ analytical column (Polaris C18-A, $180 \AA, 3 \mu \mathrm{m}$ ). An aliquot of sample was loaded onto the trap column at $2 \mu \mathrm{L} \cdot \mathrm{min}^{-1}$ in $0.1 \%(v / v)$ FA. The peptides were then eluted during a 100-min non-linear gradient of acetonitrile (from 3\% to $50 \% v / v$ ) in $0.1 \%(v / v)$ FA at $0.4 \mu \mathrm{L} \cdot \mathrm{min}^{-1}$. The mass spectrometer ran in positive ion mode and MS scans were acquired over a range from 300 to 3000 mass-to-charge ratio $(\mathrm{m} / \mathrm{z})$ at 4 spectra $\cdot \mathrm{s}^{-1}$. Precursor ions were selected by auto-MS/MS with a maximum of 4 precursors per cycle and active exclusion set at 2 spectra for $0.1 \mathrm{~min}$.

Analysis of MS/MS spectra were performed by Spectrum Mill MS Proteomics Workbench (Rev B.04.00.127; Agilent Technologies). Cysteine carbamidomethylation and methionine oxidation were used as fixed and variable modifications, admitting 2 tryptic missed cleavages per peptide. The search was conducted against the database of Zea mays (ID 4577) protein sequences (Aug 2017, 130162 entries) downloaded from UniProtKB/Swiss-Prot (http:/ / www.uniprot.org/), and concatenated with the reverse one. The threshold used for protein identification was false discovery rate (FDR) $\leq 1 \%$, number of unique peptides (NUP) $\geq 2$, protein amino acid coverage $\geq 5 \%$ if NUP $<4$. Peptide quantification was obtained as the spectrum intensity (SI) of the precursor $\left(\mathrm{MH}^{+}\right)$. Protein quantification was obtained summing the SI of all the identified peptides in the protein. Protein abundance was normalized as the \% with respect to the abundance of all validated proteins in the sample (\%SI). Proteins showing at least a two-fold change in their \%SI among at least 2 of all the experimental conditions were analysed according to the two-way ANOVA test to ascertain the source of variations and then by one-way ANOVA to assess the significance of the differences (Tukey post hoc, $p<0.05$ ).

Supplementary Materials: Supplementary materials can be found at http://www.mdpi.com/1422-0067/19/8/ 2202/s1. Supplementary Data 1. Comparative proteomic profiles of maize plants. The file reports data about the comparative characterization of the root (Supplementary Table S1) and leaf (Supplementary Table S2) proteomes. Supplementary Data 2. Changes in the level of proteins differentially accumulated in roots and leaves of maize plants. The file reports as bar charts the levels of the proteins differentially accumulated in roots and leaves of maize plants during the different nutritional treatments.

Author Contributions: Conceptualization, B.P. and L.E.; Formal analysis, B.P.; Investigation, B.P. and L.E.; Validation, B.P. and L.E.; Visualization, B.P.; Writing—original draft, B.P.; Writing—review \& editing, B.P.

Funding: This work was supported by grant Finanziamento delle attività base di ricerca from the Italian Ministry for Education, University and Research (MUIR) assigned to Dr. Bhakti Prinsi.

Acknowledgments: The authors thank Lesley Currah for polishing the English in the manuscript.

Conflicts of Interest: The authors declare no conflict of interest. 


\section{Abbreviations}

$\begin{array}{ll}\text { 2-ME } & \text { 2-Mercaptoethanol } \\ \text { CBB } & \text { Coomassie Brilliant Blue } \\ \text { DPs } & \text { Differentially abundant Proteins } \\ \text { FA } & \text { Formic acid } \\ \text { NUP } & \text { Number of unique peptides } \\ \text { OPA } & o \text {-Phthalaldehyde } \\ \text { SDS } & \text { Sodium dodecyl sulfate } \\ \text { SI } & \text { Spectrum intensity }\end{array}$

\section{References}

1. Hawkesford, M.; Horst, W.; Kichey, T.; Lambers, H.; Schjoerring, J.; Møller, I.S.; White, P. Marschner's Mineral Nutrition of Higher Plants, 3rd ed.; Elsevier/Academic Press: Amsterdam, The Netherlands, 2011; pp. 135-151. ISBN 978-0-12-384905-2.

2. Miller, A.J.; Cramer, M.D. Root nitrogen acquisition and assimilation. Plant Soil 2004, 274, 1-36. [CrossRef]

3. Andrews, M.; Raven, J.A.; Lea, P.J. Do plants need nitrate? The mechanisms by which nitrogen form affects plants. Ann. Appl. Biol. 2013, 163, 174-199. [CrossRef]

4. Krapp, A. Plant nitrogen assimilation and its regulation: A complex puzzle with missing pieces. Curr. Opin. Plant Biol. 2015, 25, 115-122. [CrossRef] [PubMed]

5. Kiba, T.; Krapp, A. Plant nitrogen acquisition under low availability: Regulation of uptake and root architecture. Plant Cell Physiol. 2016, 57, 707-714. [CrossRef] [PubMed]

6. Nunes-Nesi, A.; Fernie, A.R.; Stitt, M. Metabolic and signaling aspects underpinning the regulation of plant carbon nitrogen interactions. Mol. Plant 2010, 3, 973-996. [CrossRef] [PubMed]

7. Masakapalli, S.K.; Kruger, N.J.; Ratcliffe, R.G. The metabolic flux phenotype of heterotrophic Arabidopsis cells reveals a complex response to changes in nitrogen supply. Plant J. 2013, 74, 569-582. [CrossRef] [PubMed]

8. Andrews, M.; Lea, P.J.; Raven, J.A.; Azevedo, R.A. Nitrogen use efficiency. 3. Nitrogen fixation. Genes and costs. Ann. Appl. Biol. 2009, 155, 1-13. [CrossRef]

9. Meyer, C.; Stitt, M. Nitrate reduction and signaling. In Plant Nitrogen; Lea, P.J., Morot-Gaudry, J.F., Eds.; Springer: Berlin, Germany, 2001; pp. 37-59. ISBN 3-540-67799-2.

10. Britto, D.T.; Kronzucker, H.J. $\mathrm{NH}_{4}{ }^{+}$toxicity in higher plants: A critical review. J. Plant Physiol. 2002, 159, 567-584. [CrossRef]

11. Esteban, R.; Ariz, I.; Crus, C.; Moran, J.F. Review: Mechanisms of ammonium toxicity and the quest for tolerance. Plant Sci. 2016, 248, 92-101. [CrossRef] [PubMed]

12. Touraine, B. Nitrate uptake by roots-Transporters and root development. In Nitrogen Acquisition and Assimilation in Higher Plants; Amâncio, S., Stulen, I., Eds.; Kluwer Academic Publishers: Dordrecht, The Netherlands, 2004; pp. 1-34. ISBN 1-4020-2727-3.

13. Hachiya, T.; Sakakibara, H. Interactions between nitrate and ammonium in their uptake, allocation, assimilation, and signalling in plants. J. Exp. Bot. 2017, 68, 2501-2512. [CrossRef] [PubMed]

14. O’Brien, J.A.; Vega, A.; Bouguyon, E.; Krouk, G.; Gojon, A.; Coruzzi, G.; Gutiérrez, R.A. Nitrate transport, sensing, and responses in plants. Mol. Plant 2016, 9, 837-856. [CrossRef] [PubMed]

15. Hirel, B.; Le Gouis, J.; Ney, B.; Gallais, A. The challenge of improving nitrogen use efficiency in crop plants: Towards a more central role for genetic variability and quantitative genetics within integrated approaches. J. Exp. Bot. 2007, 58, 2369-2387. [CrossRef] [PubMed]

16. Liu, J.; Han, L.; Chen, F.; Bao, J.; Zhang, F.; Mi, G. Microarray analysis reveals early responsive genes possibly involved in localized nitrate stimulation of lateral root development in maize (Zea mays L.). Plant Sci. 2008, 175, 272-282. [CrossRef]

17. Zamboni, A.; Astolfi, S.; Zuchi, S.; Pii, Y.; Guardini, K.; Tononi, P.; Varanini, Z. Nitrate induction triggers different transcriptional changes in a high and a low nitrogen use efficiency maize inbred line. J. Integr. Plant Biol. 2014, 56, 1080-1094. [CrossRef] [PubMed]

18. Pii, Y.; Alessandrini, M.; Dall'Osto, L.; Guardini, K.; Prinsi, B.; Espen, L.; Zamboni, A.; Varanini, Z. Time-resolved investigation of molecular components involved in the induction of $\mathrm{NO}_{3}{ }^{-}$High Affinity Transport system in maize roots. Front. Plant Sci. 2016, 7, 1657. [CrossRef] [PubMed] 
19. Amiour, N.; Imbaud, S.; Clément, G.; Agier, N.; Zivy, M.; Valot, B.; Balliau, T.; Armengaud, P.; Quilleré, I.; Cañas, R.; et al. The use of metabolomics integrated with transcriptomic and proteomic studies for identifying key steps involved in the control of nitrogen metabolism in crops such as maize. J. Exp. Bot. 2012, 63, 5017-5033. [CrossRef] [PubMed]

20. Prinsi, B.; Negri, A.S.; Pesaresi, P.; Cocucci, M.; Espen, L. Evaluation of protein pattern changes in roots and leaves of Zea mays plants in response to nitrate availability by two-dimensional gel electrophoresis analysis. BMC Plant Biol. 2009, 9, 113. [CrossRef] [PubMed]

21. Plénet, D.; Lemaire, G. Relationships between dynamics of nitrogen uptake and dry matter accumulation in maize crops. Determination of critical N concentration. Plant Soil 2000, 216, 65-82. [CrossRef]

22. Schortemeyer, M.; Stamp, P.; Feil, B. Ammonium tolerance and carbohydrate status in maize cultivars. Ann. Bot. 1997, 79, 25-30. [CrossRef]

23. Andrews, M.; Raven, J.A.; Sprent, J.I. Environmental effects on dry matter partitioning between shoot and root of crop plants: Relations with growth and shoot protein concentration. Ann. Appl. Biol. 2001, 138, 57-68. [CrossRef]

24. Cramer, M.D.; Lewis, O.A.M. The influence of $\mathrm{NO}_{3}{ }^{-}$and $\mathrm{NH}_{4}{ }^{+}$on the carbon and nitrogen partitioning characteristics of wheat (Triticum aestivum L.) and maize (Zea mays L.) plants. Plant Soil 1993, 154, 289-300. [CrossRef]

25. Kronzucker, H.J.; Glass, A.D.M.; Siddiqi, M.Y. Inhibition of nitrate uptake by ammonium in barley. Analysis of the component fluxes. Plant Physiol. 1999, 120, 283-291. [CrossRef] [PubMed]

26. Cerezo, M.; Tillard, P.; Filleur, S.; Muños, S.; Daniel-Vedele, F.; Gojon, A. Major alterations of the regulation of root $\mathrm{NO}_{3}{ }^{-}$uptake are associated with the mutation of Nrt2.1 and Nrt2.2 genes in Arabidopsis. Plant Physiol. 2001, 127, 262-271. [CrossRef] [PubMed]

27. Prinsi, B.; Espen, L. Mineral nitrogen sources differently affect root glutamine synthetase isoforms and amino acid balance among organs in maize. BMC Plant Biol. 2015, 15, 96. [CrossRef] [PubMed]

28. Gilmore, J.M.; Washburn, M.P. Advances in shotgun proteomics and the analysis of membrane proteomes. J. Proteom. 2010, 73, 2078-2091. [CrossRef] [PubMed]

29. Wang, R.; Okamoto, M.; Xing, X.; Crawford, N.M. Microarray analysis of the nitrate response in Arabidopsis roots and shoots reveals over 1000 rapidly responding genes and new linkages to glucose, trehalose-6-phosphate, iron and sulphate metabolism. Plant Physiol. 2003, 132, 556-567. [CrossRef] [PubMed]

30. Patterson, K.; Cakmak, T.; Cooper, A.; Larger, I.; Rasmusson, A.G.; Escobar, M.A. Distinct signalling pathways and transcriptome response signatures differentiate ammonium- and nitrate-supplied plants. Plant Cell Environ. 2010, 33, 1486-1501. [CrossRef] [PubMed]

31. Suzuki, A.; Oaks, A.; Jacquot, J.-P.; Vidal, J.; Gadal, P. An electron transport system in maize roots for reactions of glutamate synthase and nitrite reductase. Plant Physiol. 1985, 78, 374-378. [CrossRef] [PubMed]

32. Redinbaugh, M.G.; Campbell, W.H. Higher plant responses to environmental nitrate. Physiol. Plant. 1991, 82, 640-650. [CrossRef]

33. Cren, M.; Hirel, B. Glutamine synthetase in higher plants: Regulation of gene and protein expression from the organ to cell. Plant Cell Physiol. 1999, 40, 1187-1193. [CrossRef]

34. Lea, P.J.; Sodek, L.; Parry, M.A.J.; Shewry, P.R.; Halford, N.G. Asparagine in plants. Ann. Appl. Biol. 2007, 150, 1-26. [CrossRef]

35. Fritz, C.; Palacios-Rojas, N.; Feil, R.; Stitt, M. Regulation of secondary metabolism by the carbon-nitrogen status in tobacco: Nitrate inhibits large sectors of phenylpropanoid metabolism. Plant J. 2006, 46, 533-548. [CrossRef] [PubMed]

36. Amunts, A.; Nelson, N. Functional organization of a plant Photosystem I: Evolution of a highly efficient photochemical machine. Plant Physiol. Biochem. 2008, 46, 228-237. [CrossRef] [PubMed]

37. Allahverdiyeva, Y.; Suorsa, M.; Rossi, F.; Pavesi, A.; Kater, M.M.; Antonacci, A.; Tadini, L.; Pribil, M.; Schneider, A.; Wanner, G.; et al. Arabidopsis plants lacking PsbQ and PsbR subunits of the oxygen-evolving complex show altered PSII super-complex organization and short-term adaptive mechanisms. Plant J. 2013, 75, 671-684. [CrossRef] [PubMed]

38. Wollman, F.-A. State transitions reveal the dynamics and flexibility of the photosynthetic apparatus. EMBO J. 2001, 20, 3623-3630. [CrossRef] [PubMed] 
39. Chen, Y.-B.; Lu, T.-C.; Wang, H.-X.; Shen, J.; Bu, T.-T.; Chao, Q.; Gao, Z.-F.; Zhu, X.-G.; Wang, Y.-F.; Wang, B.-C. Posttranslational modification of maize chloroplast pyruvate orthophosphate dikinase reveals the precise regulatory mechanism of its enzymatic activity. Plant Physiol. 2014, 165, 534-549. [CrossRef] [PubMed]

40. Flügge, U.-I. Phosphate translocators in plastids. Annu. Rev. Plant Physiol. Plant Mol. Biol. 1999, 50, $27-45$. [CrossRef] [PubMed]

41. Hammargren, J.; Sundström, J.; Johansson, M.; Bergman, P.; Knorpp, C. On the phylogeny, expression and targeting of plant nucleoside diphosphate kinases. Physiol. Plant. 2007, 129, 79-89. [CrossRef]

42. Redinbaugh, M.G.; Campbell, W.H. Nitrate regulation of the oxidative pentose phosphate pathway in maize (Zea mays L.) root plastids: Induction of 6-phosphogluconate dehydrogenase activity, protein and transcript levels. Plant Sci. 1998, 134, 129-140. [CrossRef]

43. Esposito, S.; Guerriero, G.; Vona, V.; Di Martino Rigano, V.; Carfagna, S.; Rigano, C. Glutamate synthase activities and protein changes in relation to nitrogen nutrition in barley: The dependence on different plastidic glucose-6P dehydrogenase isoforms. J. Exp. Bot. 2005, 56, 55-64. [CrossRef] [PubMed]

44. Fernie, A.R.; Carrari, F.; Sweetlove, L.J. Respiratory metabolism: Glycolysis, the TCA cycle and mitochondrial electron transport. Curr. Opin. Plant Biol. 2004, 7, 254-261. [CrossRef] [PubMed]

45. Britto, D.T.; Kronzucker, H.J. Nitrogen acquisition, PEP carboxylase, and cellular pH homeostasis: New views on old paradigms. Plant Cell Environ. 2005, 28, 1396-1409. [CrossRef]

46. Coskun, D.; Britto, D.V.; Kronzucker, H.J. The nitrogen-potassium intersections: Membranes, metabolism, and mechanism. Plant Cell Environ. 2017, 40, 2029-2041. [CrossRef] [PubMed]

47. Coskun, D.; Britto, D.T.; Li, M.; Oh, S.; Kronzucker, H.J. Capacity and plasticity of potassium channels and high-affinity transporters in roots of barley and Arabidopsis. Plant Physiol. 2013, 162, 496-511. [CrossRef] [PubMed]

48. Maurel, C.; Verdoucq, L.; Luu, D.-T.; Santoni, V. Plant aquaporins: Membrane channels with multiple integrated functions. Annu. Rev. Plant Biol. 2008, 59, 595-624. [CrossRef] [PubMed]

49. Gorska, A.; Zwieniecka, A.; Holbrook, N.M.; Zwieniecki, M.A. Nitrate induction of root hydraulic conductivity in maize is not correlated with aquaporin expression. Planta 2008, 228, 989-998. [CrossRef] [PubMed]

50. Loqué, D.; Ludewig, U.; Yuan, L.; von Wirén, N. Tonoplast intrinsic proteins AtTIP2;1 and AtTIP2;3 facilitate $\mathrm{NH}_{3}$ transport into the vacuole. Plant Physiol. 2005, 137, 671-680. [CrossRef] [PubMed]

51. Stiefel, V.; Ruiz-Avila, L.; Raz, R.; Pilar Vallés, M.; Gómez, J.; Pagés, M.; Martinez-Izquierdo, J.A.; Ludevid, M.D.; Langdale, J.A.; Nelson, T.; Puigdomènech, P. Expression of a maize cell wall hydroxyproline-rich glycoprotein gene in early leaf and root vascular differentiation. Plant Cell 1990, 2, 785-793. [CrossRef] [PubMed]

52. Held, B.M.; Wang, H.; John, I.; Syrkin Wurtele, E.S.; Colbert, J.T. An mRNA putatively coding for an $O$-methyltransferase accumulates preferentially in maize roots and is located predominantly in the region of the endodermis. Plant Physiol. 1993, 102, 1001-1008. [CrossRef] [PubMed]

53. Scheible, W.R.; Morcuende, R.; Czechowski, T.; Fritz, C.; Osuna, D.; Palacio-Rojas, N.; Schindelasch, D.; Thimm, O.; Udvardi, M.K.; Stitt, M. Genome-wide reprogramming of primary and secondary metabolism, protein synthesis, cellular growth processes, ant the regulatory infrastructure of Arabidopsis in response to nitrogen. Plant Physiol. 2004, 136, 2483-2499. [CrossRef] [PubMed]

54. Barakat, A.; Szick-Miranda, K.; Chang, I.-F.; Guyot, R.; Blanc, G.; Cooke, R.; Delseny, M.; Bailey-Serres, J. The organization of cytoplasmic ribosomal protein genes in the Arabidopsis genome. Plant Physiol. 2001, 127, $398-415$. [CrossRef] [PubMed]

55. Sormani, R.; Masclaux-Daubresse, C.; Daniel-Vedele, F.; Chardon, F. Transcriptional regulation of ribosome components are determined by stress according to cellular compartment in Arabidopsis thaliana. PLoS ONE 2011, 6, e28070. [CrossRef] [PubMed]

56. Carroll, A.J. The Arabidopsis cytosolic ribosomal proteome: From form to function. Front. Plant Sci. 2013, 4, 32. [CrossRef] [PubMed]

57. Schiene, C.; Fischer, G. Enzymes that catalyse the restructuring of proteins. Curr. Opin. Struct. Biol. 2000, 10, 40-45. [CrossRef]

58. Marivet, J.; Frenso, P.; Burkard, G. Effects of abiotic stresses on cyclophilin gene expression in maize and bean and sequence analysis of bean cyclophilin cDNA. Plant Sci. 1992, 84, 171-178. [CrossRef] 
59. Lee, J.-P.; Palfrey, H.C.; Bindokas, V.P.; Ghadge, G.D.; Ma, L.; Miller, R.J.; Roos, R.P. The role of immunophilins in mutant superoxide dismutase-1-linked familial amylotrophic lateral sclerosis. Proc. Natl. Acad. Sci. USA 1999, 96, 3251-3256. [CrossRef] [PubMed]

60. Colas des Francs-Small, C.; Ambard-Breteville, F.; Small, F.; Rémy, R. Identification of a major soluble protein in mitochondria from nonphotosynthetic tissues as NAD-dependent formate dehydrogenase. Plant Physiol. 1993, 102, 1171-1177. [CrossRef] [PubMed]

61. Hanson, A.D.; Gage, D.A.; Shachar-Hill, Y. Plant one-carbon metabolism and its engineering. Trends Plant Sci. 2000, 5, 206-213. [CrossRef]

62. Lou, H.Q.; Gong, Y.L.; Fan, W.; Xu, J.M.; Liu, Y.; Cao, M.J.; Wang, M.-H.; Yang, J.L.; Zheng, S.J. A formate dehydrogenase confers tolerance to aluminum and low pH. Plant Physiol. 2016, 171, 294-305. [CrossRef] [PubMed]

63. Porta, H.; Rocha-Sosa, M. Plant lipoxygenases. Physiological and molecular features. Plant Physiol. 2002, 130, $15-21$. [CrossRef] [PubMed]

64. Shigeto, J.; Tsutsumi, Y. Diverse functions and reactions of class III peroxidases. New Phytol. 2016, 209, $1395-1402$. [CrossRef] [PubMed]

65. Cataldo, D.A.; Maroon, M.; Schrader, L.E.; Youngs, V.L. Rapid colorimetric determination of nitrate in plant tissue by nitration of salicylic acid. Commun. Soil Sci. Plant Anal. 1975, 6, 71-80. [CrossRef]

66. Coskun, D.; Britto, D.T.; Jean, Y.-K.; Schulze, L.M.; Backer, A.; Kronzucker, H.J. Silver ions disrupt K ${ }^{+}$ homeostasis and cellular integrity in intact barley (Hordeum vulgare L.) roots. J. Exp. Bot. 2012, 63, 151-162. [CrossRef] [PubMed]

67. Moore, S.; Stein, W.H. A modified ninhydrin reagent for the photometric determination of amino acids and related compounds. J. Biol. Chem. 1954, 211, 907-913. [PubMed]

68. Nelson, N. A photometric adaptation of the Somogy method for the determination of glucose. J. Biol. Chem 1944, 153, 375-380.

69. Mariotti, F.; Tomé, D.; Patureau Mirand, P. Converting nitrogen into protein-Beyond 6.25 and Jones' factors. Crit. Rev. Food Sci. 2008, 48, 177-184. [CrossRef] [PubMed]

70. Laemmli, U.K. Cleavage of structural proteins during the assembly of the head of bacteriophage T4. Nature 1970, 227, 680-685. [CrossRef] [PubMed] 